\title{
Quantumbit Cosmology Explains Effects of Rotation Curves of Galaxies
}

\author{
Thomas Görnitz ${ }^{1}$ (D) - Uwe Schomäcker ${ }^{2}$
}

Accepted: 20 June 2021 / Published online: 2 August 2021

(c) The Author(s) 2021

\begin{abstract}
Some terms identify enigmata of today's cosmology: "Inflation" is expected to explain the homogeneity and isotropy of the cosmic background. The repulsive force of a "dark energy" shall prevent a re-collapse of the cosmos. The additional gravitational effect of a "dark matter" was originally supposed to explain the deviations of the rotation curves of the galaxies from Kepler's laws. Adopting a theory founded on the core notion of absolute quantum information-Protyposis-being a cosmological concept from the outset, the observed phenomena can be explained without postulating further unknown specific "particles" or "fields". Moreover, this theory allows for a rationalization of the fact that huge black holes with their enormous jet structures, acting as "seeds" of the galaxies, are detected ever closer to the big bang. The problem of the rotation curves in the galaxies can be addressed outside of General Relativity within a Newtonian approximation: by an attenuation of the gravitational acceleration as in the modified Newtonian dynamics, or by the effect of additional invisible "particles of dark matter", yet unknown and not yet established in natural sciences. Within the Protyposis theory, these problems are solved without having to invent a lot of parameters. The cosmology of the Protyposis causes the change of the gravitational acceleration in the vicinity of large (black hole) masses and, at the same time, avoids a recollapse of the cosmos for which a cosmological constant or "dark energy" was invented.
\end{abstract}

Keywords Cosmology · Dark matter · Black holes · Quantum information · Protyposis · Dark energy

\section{Introductory Remarks}

In the CERN-Courier of Nov/Dec 2020, ${ }^{1}$ a conference report came to the conclusion:»Despite excellent advances in Higgs-sector measurements, searches for WIMP dark matter and exploration of very rare processes in the flavour realm, however, no

\footnotetext{
1 CERN-Courier, 2020 NovDec p. 21.
}

Thomas Görnitz

goernitz@em.uni-frankfurt.de

1 FB Physik, J. W. Goethe-Universität Frankfurt/M, 60438 Frankfurt am Main, Germany

2 Institut Für Mathematische Physik, TU Braunschweig, 38106 Braunschweig, Germany 
unambiguous signals of new fundamental physics have been seen.« And the next article of Beacham, de Roeck and Lanfranchi (2020) started with the statement:»The nature of dark matter (DM) remains one of the most intriguing unsolved questions of modern physics.«

The many models that shall explain the dark matter have numerous free parameters. If nonetheless, no success can be achieved, it seems to be obvious that reflections about basics are needed.

It is about "explaining"-what does this mean?

Explaining means to construct or reconstruct complex structures from simple ones. In quantum theory, simple structures are combined to complex structures by forming tensor products of the state spaces.

Currently, the most complex structures to work with are quantum fields. In the FockRepresentation, they appear as a (possibly infinite) number of quantum particles. A particle is thus simpler than a quantum field.

For two millennia, there has been the idea in physics that the way to the simple one is the way to spatially small things, to ever smaller particles. This idea was successful up to the atoms, i.e. as long as only electromagnetic interaction and gravitation needed to be considered. But then, reflective thinking showed that the "smallest particles as basis of reality" were a blind alley. Planck's formula $\boldsymbol{E}=\boldsymbol{h} \boldsymbol{c} / \boldsymbol{\lambda}$ makes it intelligible that the smaller the postulated and experimentally produced particles are, the faster they decompose and the more complex the related theories become.

It is implausible that an ever-increasing concentration of energy shall lead to simpler and simpler structures, and it contradicts the experimental and theoretical experience.

\subsection{Absolute Quantum Bits of Information (AQIs) of the Protyposis}

For mathematical reasons, the simplest state space in Quantum Theory is $\boldsymbol{C}^{2}$ - the twodimensional complex Hilbert space. The related physical structures are called 'spins' and also 'quantum bits'. However, the spins are inseparably linked to a quantum particle, and quantum particles with their infinitely dimensional state space are not 'simple'.

The simplest of the mathematically possible quantum physical structures have a structure like quantum bits. Quantum bits are mostly identified with 'information', i.e. with 'meaningful information'. 'Meaning', however, comprises a great portion of subjectivity, and subjectivity is nothing for a science that is geared to objectivity like physics.

Therefore, quantum bits must be thought of-for the theory presented here-as absolute and still free of any concrete specific meaning ("Bedeutung"). So, it became necessary to introduce a new term. The designation "Protyposis" was selected for the number of absolute and abstract bits of quantum information-AQIs-to avoid incorrect associations, e.g. with "knowledge" or some sto approximate objects by high-dimensional representationsubjective "meaning".

Whereas an AQI has a two-dimensional state space, a quantum particle has a countably infinite and a quantum field an uncountably infinite dimensional state space (Figs. 1, 2, 3, $4,5)$.

The main postulate of the protyposis is that all quantum particles and fields can be constructed from the really simplest quantum structures. However, not all AQI structures must form quantum particles or fields. 
It is possible that there are local density concentrations of AQIs in the order of galaxies and galaxy clusters that cannot be described as "consisting of particles", but which produce gravitative effects on themselves and within their surroundings.

Based on the AQIs, all objects shall be constructed that are described by physics. Any state space can be constructed as subspace of sufficiently large tensor product of $\boldsymbol{C}^{2}$ spaces. Thus, it must be possible to approximate objects by high-dimensional representations of symmetry groups, for which AQIs can be used as basis. This has been demonstrated by Görnitz et al. 1992; Görnitz and Schomäcker (2012), for example, for irreducible representations of the Poincaré group, i.e. for particles with and without mass.

Our physical concept of an orientation from particles as basis of physics towards the actually simplest quantum structures, the AQIs, requires a change in thinking-as sometimes found in natural science-like the change from geocentrism to heliocentrism.

Since previous publications for the concept presented here are not readily available at once, and since you cannot expect that these prior articles are read in conjunction with a paper that basically questions the prevailing opinion in some points, some summarizing explanations might be helpful, ahead of the proper text.

The symmetry group of a single AQI leaving the norm in its state space $\boldsymbol{C}^{2}$ invariant comprises a phase factor $\mathrm{e}^{\mathrm{i} \varphi}$, an $\boldsymbol{U}(\mathbf{1})$, and the $\boldsymbol{S U}(2)$. The matrixes of the $\boldsymbol{S U}(\mathbf{2})$ group have the form

$$
\left(\begin{array}{cc}
\mathrm{a}+i b & \mathrm{c}+i d \\
-\mathrm{c}+i d & \mathrm{a}-i b
\end{array}\right)
$$

with the specific second-order condition:

$$
a^{2}+b^{2}+c^{2}+d^{2}=1
$$

(For clarification it must be pointed out that for quantum computing, a truncated form of the quantum bits is used, i.e. not the four parameters of $\boldsymbol{C}^{2}$, but the two real parameters of the Bloch sphere.)

As is generally known, all irreducible representations of the $\boldsymbol{S U}(2)$ can be formed as partial representations of regular representations. The regular representation operates in the Hilbert space of the square-integrable functions on the maximally symmetrical space of the group, i.e. on the group itself. $\boldsymbol{S U}(2)$ as its maximally homogeneous space is an $S^{3}$. Weizsäcker had hypothesized that the three-dimensionality of the space in which everything existing is can be deduced from the quantum theory of the simple alternative [Weizsäcker $(1955,1958)]$.

Because the physical structures can be constructed from AQIs, all their states can be represented as functions on this $\boldsymbol{S}^{\mathbf{3}}$. The radius of the $\boldsymbol{S}^{\mathbf{3}}$ is designated with $\boldsymbol{R}$.

An irreducible representation does not decompose into parts. An irreducible representation of $\boldsymbol{S U}(2)$ shall therefore be understood as a representation of a structure that is not treated as "consisting of particles".

Since it is not possible to test the cosmological initial and boundary conditions experimentally, you cannot do without parameters. See, for example:

»Our main analysis models four independent BOSS power spectrum datasets across two redshift bins $\left(\mathrm{z}_{\mathrm{eff}}=0.38,0.61\right)$ in flat $\Lambda \mathrm{CDM}$, marginalizing over 7 nuisance parameters for each dataset (28 in total) and varying 5 cosmological parameters $\left(\omega_{\mathrm{b}}\right.$, $\left.\omega_{\text {cdm }}, H_{0}, A_{s}, \Sigma m_{\nu}\right) \ll[\operatorname{Ivanov}(2020)]$ 


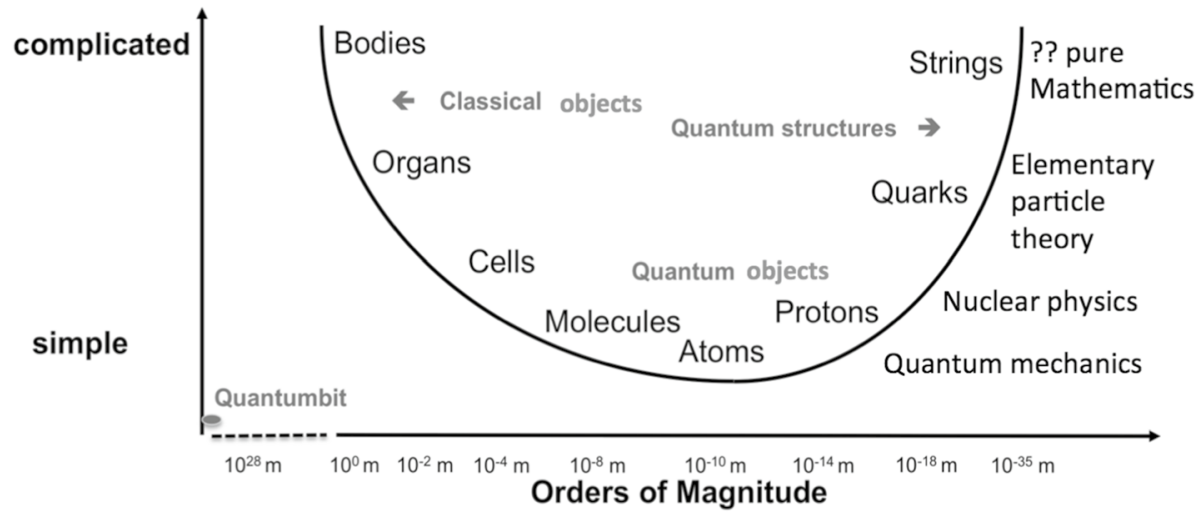

Fig. 1 When searching for simple things, the way to spatially small things proves to be a blind alley. The basic explanations must be based on the simplest structures

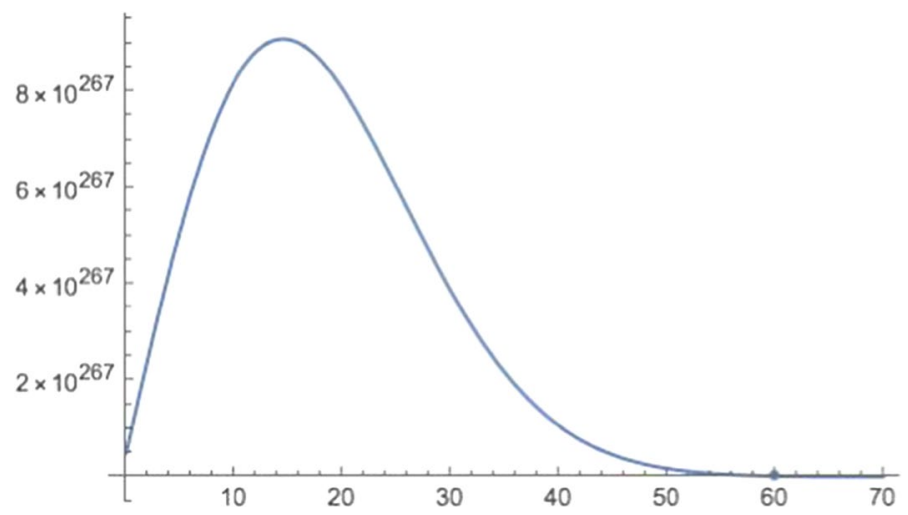

Fig. 2 For $N=900$, the curve of $f(k)$ is shown with $k=(N / 2)-j=450-j$ for the values from $k=0$ via $1 / 2 \sqrt{ } N$ and then beyond $2 \sqrt{ } N$, as an example. From the logarithmic representation it can be seen that representations beyond $2 \sqrt{ } \mathrm{N}$ can be neglected. If $\mathrm{R}$ is the radius of $\mathrm{S} 3$, then the probability to actually find a length smaller than $\mathrm{R} / 2 \sqrt{\mathrm{N}}$, is - in this example-lower than 0.00028993 and shall be neglected

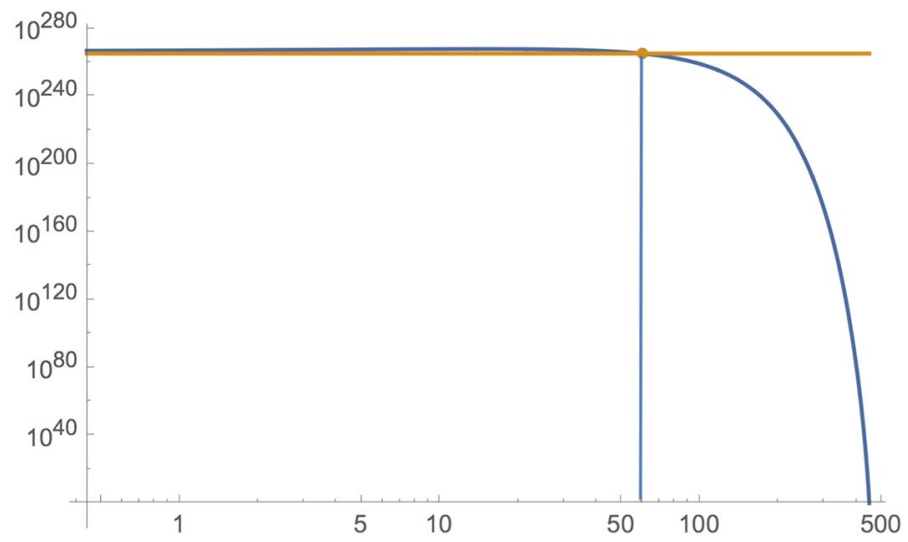

Fig. 3 For $N=900$, the curve of $f(k)$ and $f(60)$ is shown in a log-log-representation. A clear exponential decrease starts at $2 \sqrt{ } N=60$ 


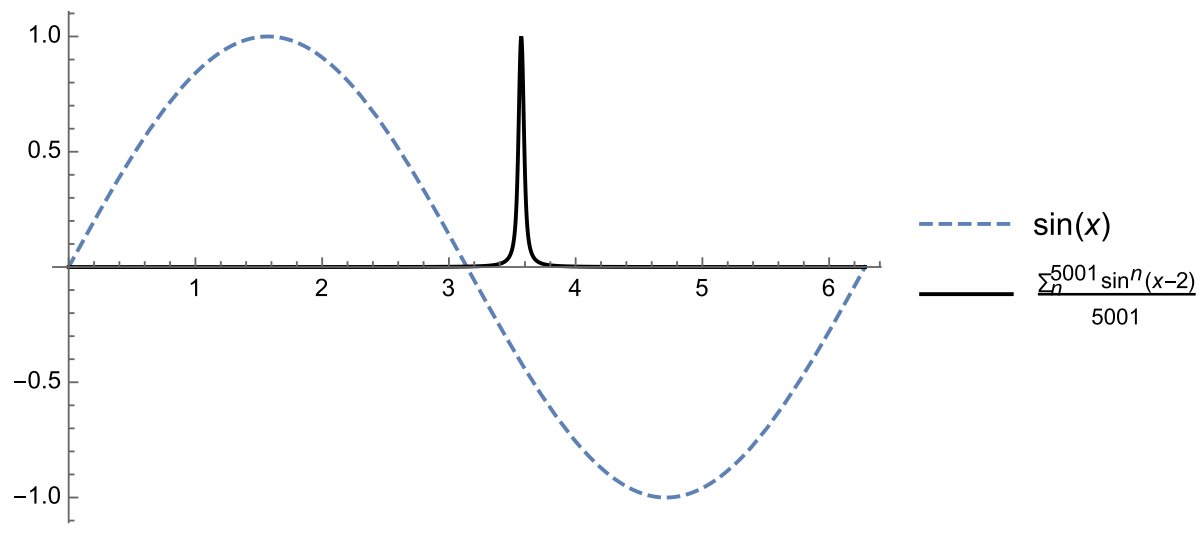

Fig. 4 The sum of sinus products can lead to an exact localization

Fig. 5 The core of galaxy NGC4261 contains a supermassive black hole with a huge jet. [https://hubblesite.org/image/82]

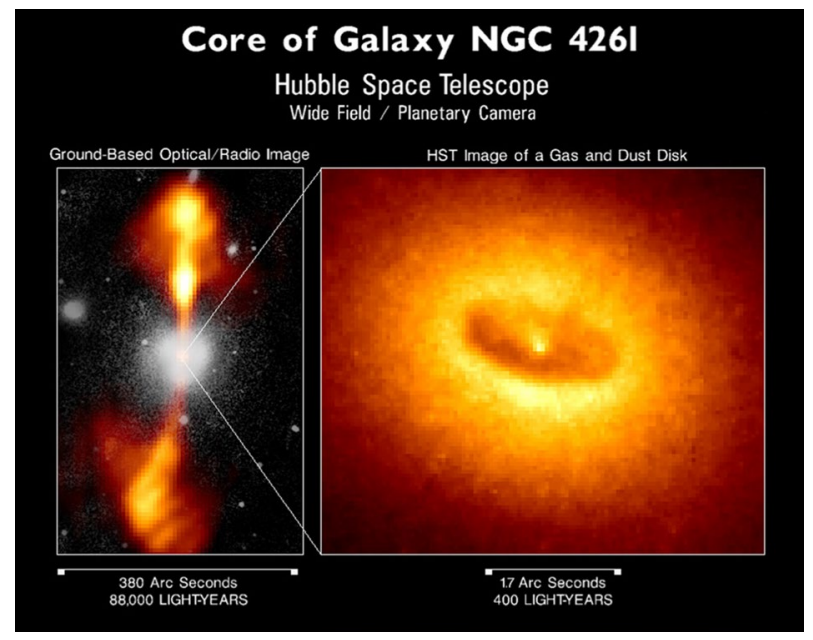

In our model, a definition of the metric as a function of the individual number of AQIs in the cosmos requires a parameter. One indication of a plausible definition comes from the area of physics where a connection of quantum theory and gravitation has already been dealt with, i.e. from the entropy of the black holes (BH). The number of quantum bits to which no concrete or specific meaning can be allocated, because they are hidden behind the horizon, which is the entropy of the $\mathrm{BH}$, is proportional to the square radius of the $\mathrm{BH}$.

Functions with only one nodal plane on $S^{3}$ belong to an AQI, i.e. a spin-1/2representation. Higher-dimensional representations contain functions with shorter wavelengths. The functions that belong to an irreducible representation ${ }^{n} \boldsymbol{D}$ with the dimension $\boldsymbol{n}$ have wavelengths of the order of $\boldsymbol{R} / \boldsymbol{n}$.

To introduce a metric on $S^{3}$, the 'smallest physically plausible length' is to be defined as a function of the number of the existing AQIs [Görnitz (1988b)]. With $N$ existing AQIs, an $N$-fold tensor product of two-dimensional spin-1/2 representations $\left({ }^{2} D_{\frac{1}{2}}\right)^{\otimes N}$ results. (In the 
following, the lower index, the "spin", is no longer indicated, but only the upper index, the dimension of representation.)

For the tensor product of $\boldsymbol{N}$ of these two-dimensional representations ${ }^{2} \boldsymbol{D}$, the following equation results for the multiplicities of the irreducible representations ${ }^{n} \boldsymbol{D}$ with the dimensions $n$ :

$$
\left({ }^{2} D\right)^{\otimes N}=\bigoplus_{\mathbf{j}=0}^{\left|\frac{N}{2}\right|} \frac{N !(N+1-2 j)}{(N+1-j) ! j !}\left|\frac{N}{2}\right|-2 \mathbf{j}+1 \text { D }
$$

with $\left|\frac{N}{2}\right|=k$ for $N=2 k$ or $N=2 k+1$

The multiplicity is defined by the function $f(j)$.

$$
f(j)=\frac{N !(N+1-2 j)}{(N+1-j) ! j !}
$$

To illustrate the behaviour of $\boldsymbol{f}(\boldsymbol{j}), \boldsymbol{N}=\mathbf{9 0 0}$ is selected as example. For this case, $\boldsymbol{j}$ goes from $\boldsymbol{0}$ to 450 . For a graphic representation, we set $\boldsymbol{k}=(\boldsymbol{N} / \mathbf{2})-\boldsymbol{j}$ and show how $\boldsymbol{f}(\boldsymbol{k})$ behaves.

From the logarithmic representation it can be seen that representations beyond $2 \sqrt{ } N$ can be neglected. If $R$ is the radius of $S^{3}$, then the probability to actually find a length smaller than $R / 2 \sqrt{ } N$, is - in this example-lower than 0.00028993 and shall be neglected.

That such a definition is not implausible is also shown by the following $\log -\log$-representation:

The following is therefore defined as smallest and thus actually achievable length, i.e. as Planck length:

$$
l_{P l}=R / 2 \sqrt{ } N_{\text {cosmos }}
$$

Conversely, this means that $\boldsymbol{R}$ with the number $\boldsymbol{N}_{\text {cosmos }}$ of AQIs increases: $\boldsymbol{R}=\boldsymbol{l}_{\boldsymbol{P l}}$ $2 \sqrt{ } N_{\text {cosmos }}$

Such a definition is not a proof, but it corresponds-except for values in the order of one-rather well to the empirical data. Like in mathematics, where nothing can be differentiated without axioms, nothing can be concluded in cosmology without certain definitions.

For further considerations, the Planck units are assumed. Then the three natural constants $\boldsymbol{h}$ and $\boldsymbol{G}$ and $\boldsymbol{c}$ - as the emphasized and highlighted velocity-receive the value 'one'.

A wavelength $\boldsymbol{R}$ can be allocated to a single AQI. According to the Planck relation $\boldsymbol{E}=\boldsymbol{h} \boldsymbol{c} / \boldsymbol{\lambda}$ the following value results for energy

$$
\mathbf{E}_{\mathrm{AQI}}=\mathbf{h} \mathbf{c} / \mathbf{R}
$$

The total energy $\boldsymbol{U}$ for $\boldsymbol{N}_{\text {cosmos }}$ of the AQIs then is

$$
\mathbf{U}=\mathbf{N}_{\operatorname{cosmos}} \mathbf{h} \mathbf{c} / \mathbf{R}=\mathbf{h} \mathbf{c} \sqrt{\mathbf{N}_{\operatorname{cosmos}}} / 2 \mathrm{l}_{\mathrm{Pl}}=\mathbf{h} \mathrm{cR} /\left(2 \mathrm{l}_{\mathrm{Pl}}\right)^{2}
$$

For a closed cosmos, there is no inflow or outlet of matter or energy. Thus, the first law of thermodynamics applies:

$$
\mathbf{d U}+\mathbf{p d V}=\mathbf{0}
$$

The volume of an $S^{3}$ is $V=\mathbf{2} \pi^{2} R^{3}$, from this it follows that

$$
d U+p d V=d R h c /\left(4 l_{P l}^{2}\right)+p 2 \pi^{2} 3 R^{2} d R=0
$$


and then

$$
+p 2 \pi^{2} 3 R^{2}=-h c /\left(4 l_{P l}^{2}\right) \text { or } p=-h c /\left(4 l_{P I}^{2} 2 \pi^{2} 3 R^{2}\right),
$$

respectively

A comparison with the energy density $\rho$

$$
\rho=\mathrm{U} / \mathrm{V}=\mathrm{h} \mathrm{c} \mathrm{R} /\left(4 \mathrm{l}_{\mathrm{PI}}^{2} 2 \pi^{2} \mathrm{R}^{3}\right)=\mathrm{hc} /\left(4 \mathrm{I}_{\mathrm{PI}}^{2} 2 \pi^{2} \mathrm{R}^{2}\right)
$$

has the following result:

$$
\mathbf{p}=-\rho / 3
$$

Now you can either claim that the velocity $c$ is distinguished because it is the expansionvelocity of the cosmos, or you insert the state equation of the AQIs from (1-10) into Einstein's equations and then obtain as exact solution an FLRW cosmos, the position-space of which expands as $\boldsymbol{S}^{\mathbf{3}}$ with $\boldsymbol{c}$.

The Protyposis can partly shape itself into matter and radiation. If you partition its energy-impulse tensor into the tensors of matter and radiation, it becomes necessary to introduce a further tensor with the structure of a vacuum or a cosmological term, however, without being constant in time. A possible, non-localized part of the Protyposis will make itself globally noticeable as "Dark Energy" in cosmology; an additional formation into "particles" is not necessary.

The partition of the energy-impulse tensor into the parts of pressureless dust, light, a cosmological term, and a possible Dark Energy/Dark Matter necessarily results as follows:

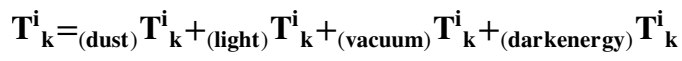

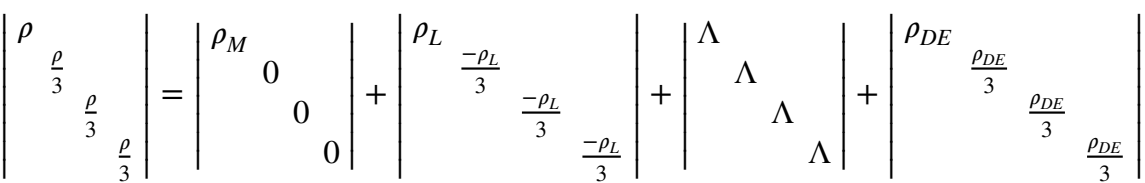

$$
\begin{aligned}
& \rho_{0} / 3 \leq \Lambda \leq \rho_{0} / 2 \leq \rho_{M}+\rho_{L} \leq 2 \rho_{0} / 3
\end{aligned}
$$

It shall be noted that this definition for the $\Lambda$ term furnishes the solution for the coincidence problem.

\section{A Look on Modern Cosmology}

The contemporary view of cosmology can be summarized as follows: The current, widely accepted $\Lambda \mathrm{CDM}$ model, despite its known shortcomings, provides a consistent description of the Universe, from its earliest beginnings to the current time. It has shown to have predictive power, a prerequisite for a theory to be considered useful.

It might be useful to briefly address these shortcomings. The empiricism problem results from the assumption of an infinitely large cosmos, which would imply that our total empirical knowledge is limited to zero \% of the entirety. For a science based on empiricismsuch as physics - this is actually a problem that cannot be ignored. There is the empirical fact-referred to as horizon problem - that the cosmic microwave background radiation is 
identical from all directions of the cosmos, whereas in many theoretical models, including the $\Lambda \mathrm{CDM}$ model, there are no satisfactory explanations for this phenomenon. To correct this problem, the "inflation" was invented, which means a hypothetical superfast inflation of the cosmos immediately after the Big Bang. Here, too, there is no scientific evidence, and, moreover, the state equation of the inflation $\boldsymbol{\mu}=\boldsymbol{- \boldsymbol { p }}$ violates important conditions necessary for sound physics. The search for "inflatons", i.e. for particles supposed to induce inflation, has been without success so far. The problem of the cosmological constant $\Lambda$ is about having to explain a magnitude that is nearly infinitesimal, but nonetheless cannot be set to zero. The attempts to calculate $\Lambda$ by means of quantum field-theoretical methods can be seen as the biggest theoretical non-success ever achieved in physics.

Two further problems can be associated with the terms "dark energy" and "dark matter". There are many publications dealing with these topics, stating that the two structures actually do exist and that at the same time nobody has an idea what they could be. The intensive search for particles of the dark matter has been failing for decades.

The often-mentioned problem that quantum theory and general theory of relativity could not be satisfactorily combined so far is not seen as a real enigma of cosmology, but rather a problem of physics in general.

The concepts of Dark Energy, Dark Matter, Inflation, and so on, each include free parameters that can be adapted to the ever-improving observation data with any accuracy desired.

More and more frequently, there has been a call for a "new physics". However, one could get the impression here that it is imperative not to abandon the trodden paths at all.

Reconsidering all this, one could ask why not just envisage the basic principles from a new perspective?

Wouldn't it be a progress in science, if the observation data could be described within a theoretical model uniting both quantum theoretical as well as relativistic arguments, a model which at the same time manages without an enormous number of free parameters?

Isaac Newton is said to have stated: "We are standing on the shoulders of giants", and, in that sense, we think that in the present situation it might be useful to have a look back into the history of physics.

In 1954, Albert Einstein concluded his book "The Meaning of Relativity" [Einstein (1954)] with the following statement:

"One can give good reasons why reality cannot at all be represented by a continuous field. From the quantum phenomena it appears to follow with certainty that a finite system of finite energy can be completely described by a finite set of numbers (quantum numbers). This does not seem to be in accordance with a continuum theory and must lead to an attempt to find a purely algebraic theory for the description of reality. But nobody knows how to obtain the basis of such a theory."

Three famous physicians searched for a theory the basis of which is algebraic, i.e. discrete.

As early as in the 1950s, Carl Friedrich v. Weizsäcker $(1955,1958)$ had searched for a foundation of the description of nature that is based on the mathematically simplest quantum-physical structures. [Weizsäcker (1958)]. Weizsäcker proposed quantized binary alternatives, "Ur-alternatives" (today: "quantum bits"). In his book "Die Einheit der Natur “ (Weizsäcker 1971, p. 361), he thus formulated the fundamental hypothesis:

"Substanz ist Form. Spezieller: Materie ist Form. Bewegung ist Form. Masse ist Information. Energie ist Information". 
("Substance is form. More specifically: matter is form. Motion is form. Mass is information. Energy is information")

Independently, David Finkelstein (1969) had developed a similar idea in the 1960s, the "space-time-code". Here, space-time was subdivided into a kind of "chessboard" composed of tiny boxes.

In 1989, with his slogan "It from Bit “, Archibald Wheeler (1989), prompted the English-speaking world to examine the discrete fundamentals of reality more closely.

These considerations reveal the common aim to create a uniform description of the empirically accessible reality, i.e. matter, forces, and information, on the basis of the simplest possible quantum-theoretical structure.

\section{Searching for a Uniform Basis}

Research in the twentieth century on the fundamentals of physics was focused on arriving at a uniform description of the forces. Quantum forces are described as gauge interactions. From the synergy of the weak and the electromagnetic interactions with their gauge groups $U(1)$ and $S U(2)$, the existence of the Higgs boson was forecasted, which eventually was found in 2012.

A unification of these two groups with $S U(3)$, the gauge group of strong interaction was supposed to be possible via the construction of increasingly larger groups, e. g., $S U(5)$ or $S O(10)$. Here, GUT (great unified theory) and $S U S Y$ (the supersymmetry) were postulated. As a consequence, many of new fundamental particles were forecasted for the LHC, the Large Hadron Collider at CERN. However, none of all those forecasted particles has yet been found.

Attempts to quantize also the General Theory of Relativity (GTR) are still going on.

The homogeneity and isotropy of the background radiation turned out to be a problem for the current standard model of cosmology. Previous models could not explain why the background radiation is identical even if coming from directions, which could-based on the model-never have had causal contact. The inflation concept invented to explain this finding has been more and more challenged in recent years. [Ijjas, (2017)].

In addition, the primordial huge black holes $(\mathrm{BH})$ around which the galaxies have formed cause major theoretical problems. [Steinhardt, (2016), Mortlock et al. (2011)].

Many new particles were postulated as underlying dark matter [Cirelli et al. (2012)] and dark energy, required in the current cosmological standard model as parameters to be adjusted. Not a single one of these forecasted particles has yet been found as a real particle. However, as shown by recent results from solid-state physics, the theoretical structures developed and their ramifications are not only of mathematical interest. The axions are one sort of these hypothetical particles. Although they do not exist as particles of dark matter in cosmos, they exist in very complex solid-state systems as quasiparticles, behaving inside the solid-similar to the phonons - as if they were such quantum particles. [Gooth et al. (2019)].

Applying the GTR to black holes results in the disappearance of the matter inside within a mathematical point. Such an infinitely high density is a non-physical result. This is another reason why there has been a call for a "new physics" for quite some time. 


\section{History and Reasons for the AQIs}

Weizsäcker $(1955,1958)$ started with the logic. He summarized his results at that time in the monograph "Aufbau der Physik" (1985). There, Weizsäcker (1985, p. 320) expressly underlines a "non-classical logic as the core of quantum theory". He recognized the quantum bits, the "Urs", as "information" in the sense of knowledge or meaning. They were referring to the relation between "two semantic levels" and thus had to be understood as being relative. v. Weizsäcker postulated: "An absolute concept of information makes no sense." (1985, p 172).

"Two semantic levels" connect the quantum bit to a meaning that results from the individual concrete context. This results in an essential subjective portion. But the subjectivity produces a discrepancy to physics that, from its target and structure, actually has to exclude subjectivity. [Hornberger, (2014)].

Therefore, quantum bits are to be understood as absolute and must be conceived as yet being free of specific meaning and hence objective.

In other words, "meaning" with its subjective aspects shuts the door to the "objectivity" of physics.

What is required are Absolute Bits of Quantum Information: AQIs. To break the automatic association of information = meaning, a new designation void of the previous associations was imperative: Protyposis.

As to Finkelstein and Wheeler, they were still guided by the millennia-old atomistic: If something shall become simpler, it must become smaller.

To this day, that idea still is the prevailing principle in quantum theory, even while in experiments indivisible quantum systems can be generated with expansions of more than $1200 \mathrm{~km}$ [Pan, (2017)], and increasingly larger molecules show interferences in the double-slit experiment. [Hornberger (2014)].

Despite these experimental manifestations of widespread quantum systems, it seems difficult to accept that the concept of "the-smaller-the-simpler" is a contradiction to the essence of quantum theory.

Quantum theory shows with $\boldsymbol{E}=\boldsymbol{h c} / \boldsymbol{\lambda}$ : the smaller, the more energy is required. This is why the accelerators used to access ever smaller spatial areas of nature need to become more and more powerful. However, that ever-higher energy will lead to ever-simpler structures does not only sound strange, but it also contradicts the experimental findings. The smaller the spatial areas generated by the accelerators become, the shorter is the time in which the created structures explode in ever more complex decay products.

This does not indicate "simplicity". It is, on the contrary, nearly trivial that by using increasingly more information, ever smaller structures can be acquired. Then, Planck's formula suddenly becomes comprehensible, and simplicity can be understood as "widespread".

It was essential to realize that the absolute quantum bit, the AQI, as the "smallest unit of information", with the potential of acquiring specific meaning, is to be connected to the lowest possible localization, i.e. being of cosmic extent.

An AQI thus has to be understood as being a cosmic structure. A possible visualization would be a "fundamental oscillation mode of the cosmic space".

Accepting information as the fundamental structure, quantum theory can more easily be understood. It is implausible that high energy will lead to the creation of something "simple", just because the created structure is confined to a small spatial region. By contrast, 
it is plausible that a large amount of information allows localization in a small region of space.

\section{How Should "Its From Bits" be Understood?}

What has so far been gained from a theory-one Einstein might have strived for-based on a discrete fundamental structure?

Starting from the mathematically simplest possible quantum structures, explanations could be given for the more complex structures examined so far in physics.

However, on that road, new concepts and imaginations were required to make advances possible.

During the half-century since v. Weizsäcker's statement, the necessary theoretical and mathematical relations between quantum theory and cosmology have successfully been advanced from their early beginnings [Görnitz, (1986), Weizsäcker (1986)], even though still off the mainstream.

In 1988, the first cosmological model was presented based on fundamental quantumtheoretical arguments, i.e. founded on a discrete basis. This model is an exact solution of the GRT with the state equation $p=-\rho / 3$, as could be deduced from these quantum-theoretical arguments. [Görnitz (1988a, b)].

Weizsäcker's considerations had established that the three-dimensional position-space could be understood as the maximum homogeneous space of the symmetry group $S U(2)$ of a quantum bit, i.e. as an $S^{3}$ hypersphere. [Weizsäcker (1985)].

All irreducible representations of the $S U(2)$ group are sub-representations of the "regular representation" which is defined in the Hilbert space of the square-integrable functions on this $S^{3}$ sphere. A single quantum bit corresponds to a spin-1/2 representation of the $S U(2)$ group. As a sub-representation of the regular representation, this representation corresponds to wave functions with the largest wavelengths, which is why the state of an AQI is represented by a wave function on the $S^{3}$ sphere with a single nodal plane.

A multitude of quantum bits corresponds to tensor products of such spin- $1 / 2$ representations. These tensor products of two-dimensional representations can be reduced to higherdimensional irreducible representations, corresponding to functions with more nodes and, thus, shorter wavelengths.

The sine function may serve to visualize how products of extended entities lead to something strongly localized.

For a long time, the prevailing idea was that a closed cosmos would have to re-collapse. In 1988, a closed cosmos expanding at the speed of light was too much ahead of the times to be appreciated, as then pressure in GRT was postulated only for the range between dust (pressure $\boldsymbol{p}=\boldsymbol{0}$ ) and light (pressure $\boldsymbol{p}=\boldsymbol{\rho} / \mathbf{3}$ ), i.e., for $\boldsymbol{0} \leq \boldsymbol{p} \leq \boldsymbol{\rho} / \mathbf{3}$. As long as pressure is seen within a particle context, this, in fact, is consistent. The notion of particles can hardly be reconciled with the idea of negative pressure. Nowadays, also the concept of a negative cosmological pressure is accepted.

The 1988 model [Görnitz (1988a, b)] solved the horizon problem without requiring an ad hoc explanation like inflation. It explained the magnitude of an effective cosmological constant $\Lambda_{\text {eff }}$, i.e., a term associated with an energy-momentum tensor analogous to the vacuum. Moreover, it made the nucleon-photon relationship plausible.

Subsequently, within the framework of the Protyposis theory and using inherent quantum-physical and thermodynamic arguments, it was demonstrated how the validity of the 
GRT can be made plausible for gravitational phenomena within the cosmos. [Görnitz (2011)].

Furthermore, a solution for the interior of black holes was deduced. Based on quantumbits, this solution avoids the Schwarzschild singularity and thus the disappearance of the matter inside the black hole within a mathematical point. [Ruhnau (1989), Görnitz (2013)] Originating from the Protyposis concept, the interior of a black hole can be seen like a strongly miniaturized version of the cosmic space.

Still in collaboration with v. Weizsäcker, an endeavor was initiated to establish the theoretical foundations for addressing the issue of how the "It" is formed from quantum bits, namely, matter in the form of relativistic particles. [Görnitz, (1992), Schomäcker (2012)] The states of such a quantum particle form an irreducible representation of the Poincare group, which can be constructed from quantum bits.

Moreover, the group structure of the three fundamental gauge interactions could be established as a consequence of the group structure of the bits. [Görnitz (2014), Schomäcker (2016)] As the Protyposis foundation allows one to reduce all entities explored in fundamental science, i.e., matter and forces, to a unifying basis, there is no longer any recognizable need to compress the forces into a hypothetic "uniform force".

In the present paper, we will demonstrate how this quantum-theoretically based cosmology can also explain effects, for which dark energy and dark matter were invented.

\section{What is the Main Difference to Previous Physics?}

One essential difference to previous theoretical explanations is that the fundamental structures of quantum theory are subjected to an in-depth reconsideration.

So far, quantum field theory has largely worked using the point particle model. We agree with Finkelstein (1969, p. 1262) who states that the idea of "points" is quite problematic:

»Each point, as Feynman once put it, has to remember with precision the values of indefinitely many fields describing indefinitely many elementary particles; has to have data inputs and outputs connected to neighboring points; has to have a little arithmetic element to satisfy the field equations; and all in all might just as well be a complete computer.«

Getting rid of a millennia-old idea, and realizing that the fundamental entities of nature are no longer to be searched in spatially smallest units, the "points", certainly is the biggest challenge for the scientific imagination presently.

However, considering that the fundamental is to be found in the smallest units of information, the principles of quantum theory become more comprehensible. Little information is simple, much information is complex. Obviously, it takes much information to achieve precise localization, and, consequently, to form spatially small structures.

"Explaining" in science, means to reconstruct a complex structure from simpler structures. The simplest quantum structure that is mathematically conceivable has a two-dimensional complex state-space, being commonly called a quantum bit.

An essential difference between the Protyposis and the technically applied quantum information (QI) is that in the latter only two real parameters (two angles) are considered, spanning the surface of the so-called Bloch sphere. This is expedient here, since the other parameters are already defined by the carrier (mostly a photon) of such a bit of QI. By 
contrast, in dealing with the fundamental issues of physics, the full two-dimensional complex space with its four real parameters must be taken into account.

The concepts of v. Weizsäcker, Finkelstein, and Wheeler essentially refer to such a basic quantum structure featuring the full two-dimensional complex state-space.

What are the consequences of the quantum-theoretical considerations for cosmology?

The approaches by v. Weizsäcker, Finkelstein and Wheeler mainly refer to such a simplest quantum structure with the full two-dimensional complex state-space.

What is the result of the quantum-theoretical considerations for the cosmology?

\section{Current Problems in Cosmology and Astrophysics}

- The $\Lambda C D M$ model requires an explanation for the magnitude of the cosmological constant $\Lambda$. (The field-theoretical result was off by very many orders of magnitude.) The current discussion involves the notions of "Dark Energy" and "Quintessence".

"Quintessence " is introduced according to $p=\omega \rho$. Here, however, $\omega<-1 / 3$ and all the more $\Lambda$ with $p=-\rho$, are in variance with essential physical energy conditions. [Hawking (1973)].

Moreover, the $\Lambda \mathrm{CDM}$ model requires "inflation" to explain the homogeneity and isotropy of the background radiation. There are, however, important arguments against "inflation". For example, Paul Steinhardt one of the inventors of inflation, realizes today that the original hopes have not been fulfilled:

»The inflationary paradigm is fundamentally untestable, and hence scientifically meaningless.« [Steinhardt (2014)]

Later on, such arguments were formulated even more clearly. [Ijjas (2017)].

- The rotation curves in the outside regions of the galaxies require an additional gravitative effect to explain the deviation of the rotational velocities from Kepler's laws. It appears that they are subject to the action of an invisible matter. Here, the term "Dark matter" came up.

For about tree decades, the search for hypothetic particles of dark matter has been unsuccessful.

- Already in early galaxies, there were huge "primordial black holes". These are to be understood as the "seeds" of the galaxies and they arose very early after the Big Bang.

The very early emergence of huge black holes is a problem:

»Indeed, there has historically been an "impossibly early black hole" problem similar to the impossibly early galaxies « [Steinhardt et al. (2016)]

With BOSS [Ivanov 2020] und BigBOSS, cosmology has entered a phase of precision cosmology.

»Both CMB (cosmic microwave background) measurements and LSS (large scale structures) data have proven to be extremely powerful in pursuing this program. « [Raveri, 2020]

On the other hand, in the history of cosmology, a very good adaptation to the data with many free parameters could be a hint, like for example in case of Ptolemy and Copernicus, to consider a change of the prevailing paradigm. 
»At the same time, hints of discrepancies between different cosmological probes within the $\Lambda \mathrm{CDM}$ model have been found. The expansion rate of the Universe as derived from the CMB differs from distance ladder measurements. Data from LSS surveys and the CMB show different pictures of how cosmological structures grew over time. Even though these discrepancies were barely note-worthy in the past, their statistical significance continues to steadily increase and might point toward the fact that we are close to a radical, paradigm-shifting discovery. « [Raveri, 2020]

With the present paper, we wish to give suggestions for an interpretation of the data that does not primarily concentrate on unknown particles.

\section{The Protyposis Cosmology}

According to v. Weizsäcker's approach, the mathematical model of the three-dimensional position-space, the cosmos, is the maximal homogeneous space of the $S U(2)$ group, i.e., the group itself. It is an $S^{3} \operatorname{cosmos}$, the three-dimensional surface of a four-dimensional sphere.

Based on representation theory for the $S U(2)$ group, a relation can be established between the cosmic curvature radius $R$ and a minimal length, identified as the Planck length $\lambda_{P l}$, for a given number $N$ of AQIs: $R \sim \lambda_{P l} \sqrt{ } N$. (Görnitz, 1988b) Accordingly, an increasing number $N$ of AQIs in the cosmos is to be interpreted as an increase of the cosmic radius.

Applying Planck's relation, $(\boldsymbol{E}=\boldsymbol{h} \boldsymbol{c} / \boldsymbol{\lambda})$, between energy $E$, Planck's constant $h$, speed of light $c$, and wave length $\lambda$, and invoking the first law of thermodynamics, the state equation for the AQIs,

$$
\mathbf{p}=-\rho / 3
$$

could be derived from the quantum-theoretical considerations. [Görnitz (1988b)].

In GTR, for a Friedmann-Lemaître-Robertson-Walker-(FLRW) cosmos, this state equation implies an expansion at the speed of light.

At a somewhat later, Wei Chen and Yong-Shi Wu, on other grounds, concluded a variable cosmological term with similar behaviour. [Chen, (1990)] Shortly thereafter, there were papers by Moncy V. John et al.[John \& Joseph, (1996, 2000), John \& Narlikar, (2002), John (2019)] promoting a model with a state equation like Eq. (8-1).

Since 2012, Fulvio Melia, together with other scientists, has been analyzing the observation data, relying on extensive examinations. He demonstrates that a cosmic expansion at $c$ is in most cases better adapted to the more recent and more precise data than the $\Lambda \mathrm{CDM}$ model — and without lots of free parameters required in the $\Lambda \mathrm{CDM}$ model [Melia \& Shevchuk, (2012), Melia \& Maier (2013), Wei, (2015), Melia \& McClintock, (2015), Melia \& López-Corredoira (2017), López-Corredoira (2016), Melia, (2016), Melia, (2017), Yennapureddya, (2018)]. The model by Melia et al., too, requires a state equation according to Eq. (8-1).

As this state equation implies, within the GTR, an expansion of the cosmos at the speed of light, inflation becomes dispensable.

As distinguished from the closed Protyposis cosmos $(k=1)$, Melia et al. mainly suppose a flat space $(k=0)$.

However, as a consequence of the $k=0$ model there is the empiricism problem (scarcely been reflected on) due to the infinitely large volume of space. Cosmologies postulating a 
flat, infinitely extended position-space capture empirically-by theory and definition—only zero percent of the postulated reality.

According to new results from the "Planck Legacy 2018 release", Aghanim N., et al. (2018) there are strong indications that a closed cosmos, as postulated in the framework of the Protyposis cosmology based on quantum-theoretical arguments, is now being substantiated by observation data. In a recent paper, somewhat melancholically titled»Planck evidence for a closed Universe and a possible crisis for cosmology«. DiValentino, Melchiorri, and Silk (2020) note that.

$»$... the Planck power spectra prefer a closed Universe ...«.

The $\Lambda \mathrm{CDM}$ model postulates an acceleration of the cosmic expansion. Some authors suspect that the motion of the local group against the cosmic background may cause such an observational effect. [Colin (2019)].

If all these different findings could further be verified and validated, a solution based on Protyposis cosmology to all of the so-called cosmological problems would be at hand.

\section{Gravitation as an Effect of the Cosmos on Internal Inhomogeneities}

Realizing that the GTR provides an excellent description of gravitation should not prevent us from reflecting on its role with regard to cosmology.

Out of the infinitely many cosmological solutions of the GTR only a single one can describe the empirically accessible cosmos. Einstein's mathematically highly interesting equations thus cover a host of entities that have no actual physical counterpart.

The fundamental quantum theory of the quantum bits made an access to the spatial structure of the cosmos possible. Assuming that the relation derived for the curvature of space and the matter-energy content of the cosmos can also be applied to local inhomogeneities, then gravitation can be understood as an effect of the cosmos on local internal inhomogeneities. [Görnitz (2011)].

Thus, the quantum-theoretical foundation of cosmology provides a rationalization why the GRT allows for such a good description of any gravitational processes within the cosmos.

Interpreting the validity of Einstein's equation in this context can help to understand the strange orbital velocities of stars in galaxies. As will be shown below cosmic influences can explain the observed orbital velocities, without having to change Newton's law with a new parameter as in MOND [Milgrom (1994)], or postulate unknown particles as with dark matter.

In these considerations, besides Planck's relation $(\boldsymbol{E}=\boldsymbol{h} \boldsymbol{c} / \boldsymbol{\lambda})$, the following physically plausible and proven hypotheses are needed:

- There is a distinguished velocity: $\boldsymbol{c}$.

- In a closed system, the first law of thermodynamics applies: » $\boldsymbol{d U}+\boldsymbol{p} \boldsymbol{d} \boldsymbol{V}=\boldsymbol{0} \ll$.

- The second law of thermodynamics refers to an increase of entropy, that is, information of which only the amount can be known, but to which no specific meaning can be attributed.

To our knowledge Theodore Jacobson (1995) was the first to observe that gravity is a phenomenon of thermodynamics. He wrote: 
$» \ldots$ the Einstein equation is an equation of state. It is born in the thermodynamic limit as a relation between thermodynamic variables, and its validity is seen to depend on the existence of local equilibrium conditions. This perspective suggests that it may be no more appropriate to quantize the Einstein equation than it would be to quantize the wave equation for sound in air.«

Subsequently, also Erik Verlinde (2011) postulated a connection between gravity and thermodynamics. However, he combined his approach with string theory and the holographic principle. Here, a bit would be associated with a very small spatial extension and, according to Planck's formula, with a very high concentration of energy. Obviously, this is the opposite of the theory presented here.

Everything within the cosmos is subject to gravitation.

This makes it understandable that the AQIs can develop-under the effect of their gravitation-local formations of higher density than in their surroundings. Primarily, this will only result in gravitational effects.

The AQIs can form quantum particles, which may be massless like photons, or have a rest mass if they carry a charge. Depending on the type of charge-leptonic, baryonic, or possibly electromagnetic - a quantum interaction is added to gravitation, i.e., the weak, strong, or possibly electromagnetic force.

\section{The Formation of Early Black Holes}

So far, no process is known that would-in the time frame according to the $\Lambda$ CDM model - allow the formation of early black holes out of any kind of gas or dust.

For example, Mortlock et al. (2011) stated:

»The existence of $\sim 10^{9} \mathrm{M}_{\odot}$ black holes at $\mathrm{z} \simeq 6$ already placed strong limits on the possible models of black-hole seed formation, accretion mechanisms and merger histories; the discovery that a $2 \times 10^{9} \mathrm{M}_{\odot}$ black hole existed just $0.77 \mathrm{Gyr}$ after the Big Bang makes these restrictions even more severe.«

As shown by Melia and McClintock (2015), Yennapureddya and Melia (2018), at least the problem of a shortage of time is solved in a cosmos expanding at $c$.

Nonetheless, there is still the difficulty that the collapsing matter would be blown away again by the emerging radiation pressure due to electromagnetic interaction. [Smith, (2019)].

»There is a limit to the rate of how fast this assembly could have occurred, first worked out by the British astrophysicist Arthur Eddington. The idea here is that the higher the mass assembly rate, the larger the output of radiation. At some point the outward pressure exerted by the radiation is able to overwhelm the inward force of gravity. This balancing of forces defines the Eddington (accretion) rate, the maximum growth rate of black holes.«

However, as long as no relativistic particles with electrical charge are formed, the condensing Protyposis is subjected to gravitative interaction only. Thus, for this form of the AQIs, the Eddington limit does not matter.

Thus, after the Big Bang, AQIs may quickly have formed very large black holes. Likewise, it is possible that high-density formations of AQIs have agglomerated extending over large areas of space, without the collapse of such clouds to black holes or to decomposition 
into relativistic particles. However, once there were also particles, galaxies with stars may form around the black holes from normal matter.

\section{The Black Hole in an Expanding FLRW Cosmos}

The structural insights enabled by the AQI concept can be applied to the problems of huge black holes around which the galaxies have formed. For example, the enormous jets in the vicinity of active black holes can also be due to gravitative causes, in addition to electromagnetic ones, as will be demonstrated in the following.

In the Protyposis theory, the metric of the cosmos has the form of an FLRW line element: [Görnitz (1988a\&b)]

$$
\left.d s_{P 1}^{2}=c^{2} d t^{2}-(1+c t)^{2}\left[\left(1-r^{2}\right)^{-1} d r^{2}+r^{2} d \Omega^{2}\right)\right]
$$

Using the transformation $1+c t=e^{(1+\tau)}$ and $r=\sin \rho$ we get $d t=d \tau e^{(1+\tau)} / c$ and $d \rho^{2}=d r^{2}\left(1-r^{2}\right)^{-1}$. Accordingly, the line element takes on the following form:

$$
\mathrm{ds}_{\mathbf{P}^{2}}=\mathrm{e}^{2(1+\tau)}\left[\mathrm{d} \tau^{2}-d \rho^{2}-\sin ^{2} \rho\left(d \theta^{2}+\sin ^{2} \theta d \varphi^{2}\right)\right]
$$

Like every FLRW cosmos, the Protyposis cosmos is homogeneous and isotropic. Therefore, the metric has to be changed if a black hole is to be described in it.

In a famous paper, Prahalad Chunnilal Vaidya (1977) has demonstrated how the metric of a closed expanding FLRW cosmos changes locally in the presence of a rotating black hole with the gravitative mass $m_{0}$ and the angular momentum $\boldsymbol{J}$, i.e. how the Kerr solution can be embedded in a variable FLRW cosmos.

Vaidya defines two parameters, the gravitative mass of the black hole $\boldsymbol{m}=\boldsymbol{G} \boldsymbol{m}_{0} / \boldsymbol{c}^{2}$, as well as its angular momentum $\boldsymbol{J}$ via $\boldsymbol{a}=\mathbf{J} / \mathbf{c m}_{\mathbf{0}}$. The metric is given with Vaidya, [Eq. (15) in Vaidya (1977)], as

$$
\begin{aligned}
\mathrm{ds}_{\mathrm{VBH}^{2}}= & \mathrm{e}^{2 \mathrm{~F}^{(\tau)}} 2\left(\mathrm{du}+\operatorname{asin}^{2 \alpha \mathrm{d} \beta}\right) d \tau-\left(1+2 m \mu e^{-2 \mathrm{~F}^{(\tau)}}\right)\left(\mathrm{du}+\operatorname{asin}^{2} \alpha \mathrm{d} \beta\right)^{2} \\
& \left.-\mathrm{M}^{2}\left[\left(1-\mathrm{a}^{2} \sin ^{2} \alpha / \mathbf{R}^{2}\right)^{-1} \mathrm{da}^{2}+\sin ^{2} \alpha \mathrm{d} \beta^{2}\right]\right\}
\end{aligned}
$$

where

$$
M^{2}=\left(R^{2}-a^{2}\right) \sin ^{2}(r / R)+a^{2} \cos ^{2} a
$$

and

$$
\mu=R \sin (r / R) \cos (r / R) / M^{2}
$$

To test whether this special form of the Vaidya metric transforms into the Protyposis metric in the absence of a black hole, the limiting value for the disappearance of mass and angular momentum must be considered.

To see what the metric becomes in the absence of the black hole, we have to set $\boldsymbol{m}=\boldsymbol{0}$ and $\boldsymbol{a}=\boldsymbol{0}$.

Then $\boldsymbol{M}^{2}$ becomes $\boldsymbol{M}_{0}{ }^{2}=\mathbf{R}^{2} \sin ^{2}(\boldsymbol{r} / \mathbf{R})$ and $\boldsymbol{\mu}_{0}=\operatorname{cotan}(\mathbf{r} / \mathbf{R}) / \mathbf{R}$.

Without loss of generality we may set $R=1$. Then the Vaidya metric becomes 


$$
\mathrm{ds}_{0}^{2}=\mathrm{e}^{2 \mathrm{~F}(\tau)}\left\{2 \mathrm{dud} \tau-\mathrm{du}^{2}-\sin ^{2} \mathrm{r}\left[\mathrm{d} \alpha^{2}+\sin ^{2} \alpha \mathrm{d} \beta^{2}\right]\right\}
$$

Using the transformation $\boldsymbol{u}=\boldsymbol{\tau}$ - $\boldsymbol{r}$ gives

$$
\mathbf{d s} s_{0}^{2}=2 \mathrm{e}^{2 \mathrm{~F}(\tau)}\left\{\mathbf{d} \tau^{2}-\mathbf{d r} \mathbf{r}^{2}-\sin ^{2} \mathbf{r}\left[\mathbf{d} \alpha^{2}+2 \sin ^{2} \alpha \mathbf{d} \boldsymbol{\beta}^{2}\right]\right\}
$$

so that with the specification

$$
\mathbf{F}(\tau)=1+\tau
$$

the cosmological Protyposis model of Eq. (11-2) is obtained.

So, it becomes apparent that by choosing the function $\boldsymbol{F}(\boldsymbol{\tau})$ this way, the Vaidya cosmology transitions into the Protyposis cosmology. This form of $\boldsymbol{F}(\boldsymbol{\tau})$ will be used in the following to comprehend the effect of a rotating $\mathrm{BH}$ in the Protyposis cosmology.

\section{Possible Jet Structures at Black Holes}

In the cores of galaxies there are often active black holes that emit huge jets. These jets can have enormous extensions, up to the range of a galaxy diameter.

In most cases, the generation of these jets is described solely by the effect of powerful magnetic fields.

A homogeneous and isotropic pressure as in Eq. (11-1) has no locally recognizable consequences. Only in the vicinity of an inhomogeneity a local effect of the cosmological pressure can become manifest.

In the gravitational field of a rotating black hole, an otherwise homogeneous and isotropic cosmological pressure varies in the direction of the rotational axis. [Vaidya (1977)] Thus, in the vicinity of a black hole the AQIs will be subject to a positive pressure in axial direction, countervailing the otherwise negative cosmological pressure according to the state equation $\boldsymbol{p}=-\boldsymbol{\rho} / 3$. This pressure can provide a purely gravitative contribution to the observed jet structures, adding to the electromagnetic contributions.

Following Vaidya, three infinitesimal, mutually orthogonal vectors can be defined in the three-dimensional space $\boldsymbol{\tau}=$ const, i.e., in the position-space at a fixed time. Here, the pressure components in axial directions are designated by $\boldsymbol{q}$, the rotationally symmetric components in direction of the rotational plane by $\boldsymbol{p}$, and the energy density by $\boldsymbol{\rho}$.

A simple toy model is used to illustrate the basic relationships between pressure and energy density in the vicinity of a black hole in an expanding cosmos, described according to the metric of Vaidya. (The model uses numerical values, which enable a simple calculation in mathematica ${ }^{\circledR}$.)

The pressure at the black hole horizon is positive. The pressure components $p$ in the direction of the plane of rotation falls very quickly to the value $\boldsymbol{p}_{\text {cosmic }}=-\boldsymbol{\rho} / \mathbf{3}$, corresponding to the cosmological environment. The pressure component $\boldsymbol{q}$ in the direction of the axis of rotation features significantly larger positive values, turning negative only at a greater distance from the horizon. So being surrounded by regions of negative cosmological pressure, that positive pressure can contribute to the jet structure observable in the quasars.

In Figs. 6, 7, we show two contour plots for the pressure components $\boldsymbol{q}$ and $\boldsymbol{p}$ and for the energy density $\rho$. It should be noted that in the GRT a solution applying to the exterior of a black hole usually is continuously extended-as in Figs. 6, 7-into the space inside the horizon down to the singular center point. As Vaidya relies entirely on GRT, quantum effects inside the horizon are not taken into account. This means that only the results 
outside the horizon are relevant. Incorporating quantum theory such a continuation through the horizon is non-physical, as has been shown long ago. [Görnitz (1989)].

Figure 8 shows the behavior of the energy density and the pressure components along two intersection lines (the same numerical values as in Fig. 6 and Fig. 7 along axis and in plane).

Approaching the black hole from outside, the originally positive cosmological energy density becomes negative near the horizon, while the originally negative cosmological pressure becomes positive.

The pressure component in the direction of the plane is many orders of magnitude smaller than that in the axis direction Fig. 9 and it adapts very quickly to the value $\boldsymbol{p}_{\text {cosmic }}=$ $-\rho / 3$.

As will be discussed below, this cosmological pressure effect can also explain the orbital velocities of the stars in the outside regions of galaxies without any additional assumptions.

The plots shown in Figs. 6, 7, 8, 9, 10 allow us to make two observations. As Vaidya's development is entirely based on the GRT, there is a continuous transition from the outer region into the interior of the horizon. However, discontinuities arise when quantum-theoretical correlations [Görnitz (2013)] are taken into account beyond the scope of the GRT. The discontinuities result from the fact that the region inside the black hole horizon represents an "impenetrable box"-with the consequence that the quantum-theoretical ground state ("vacuum") inside the "box" differs from that outside (Figs. 11, 12).

\subsection{What are the Consequences of a Black Hole Embedded in the Protyposis Cosmology?}

As shown in Fig. 10, at a greater distance from the Black Hole, the energy density $\rho$ becomes positive and the pressure components $\boldsymbol{q}$ become negative and approach the value $p_{\text {cosmic }}=-\rho / 3$.

However, in the vicinity of the Black Hole, the energy density $\rho$ and the cosmological pressure $\boldsymbol{p}_{\text {cosmic }}$ change, as well as the ratio between them.

The effect of the cosmic pressure according to $\boldsymbol{p}_{\text {cosmic }}=-\boldsymbol{\rho} / \mathbf{3}$ comes into play already very close to the Black Hole in the galactic rotational planes. This effect is essential for explaining the rotational velocities, as will be discussed in the following section.

In contrast to that, the pressure in the direction of the rotational axis is positive near the horizon. It remains positive for larger distances from the center. The difference between the positive pressure along the axis of the Black Hole and the negative cosmological pressure can therefore make a gravitational contribution to the formation of the jet structures, in addition to the magnetic effects often described.

\section{The Rotation Curves of the Galaxies}

Contrary to the behaviour along the rotational axis, the pressure remains nearly identical in the rotational plane (i.e. for the $q$ component as well as also for the $p$ component) with the cosmological pressure $\boldsymbol{p}_{\text {cosmic }}$ from formula $(8-1)$. Thus, in the rotational plane around the black hole, a pressure is quickly reached, which is only very slightly increased compared to the cosmological pressure. Based thereon, the effect can be explained, for which the dark matter has been invented, namely the flat rotational speed of galaxies. 

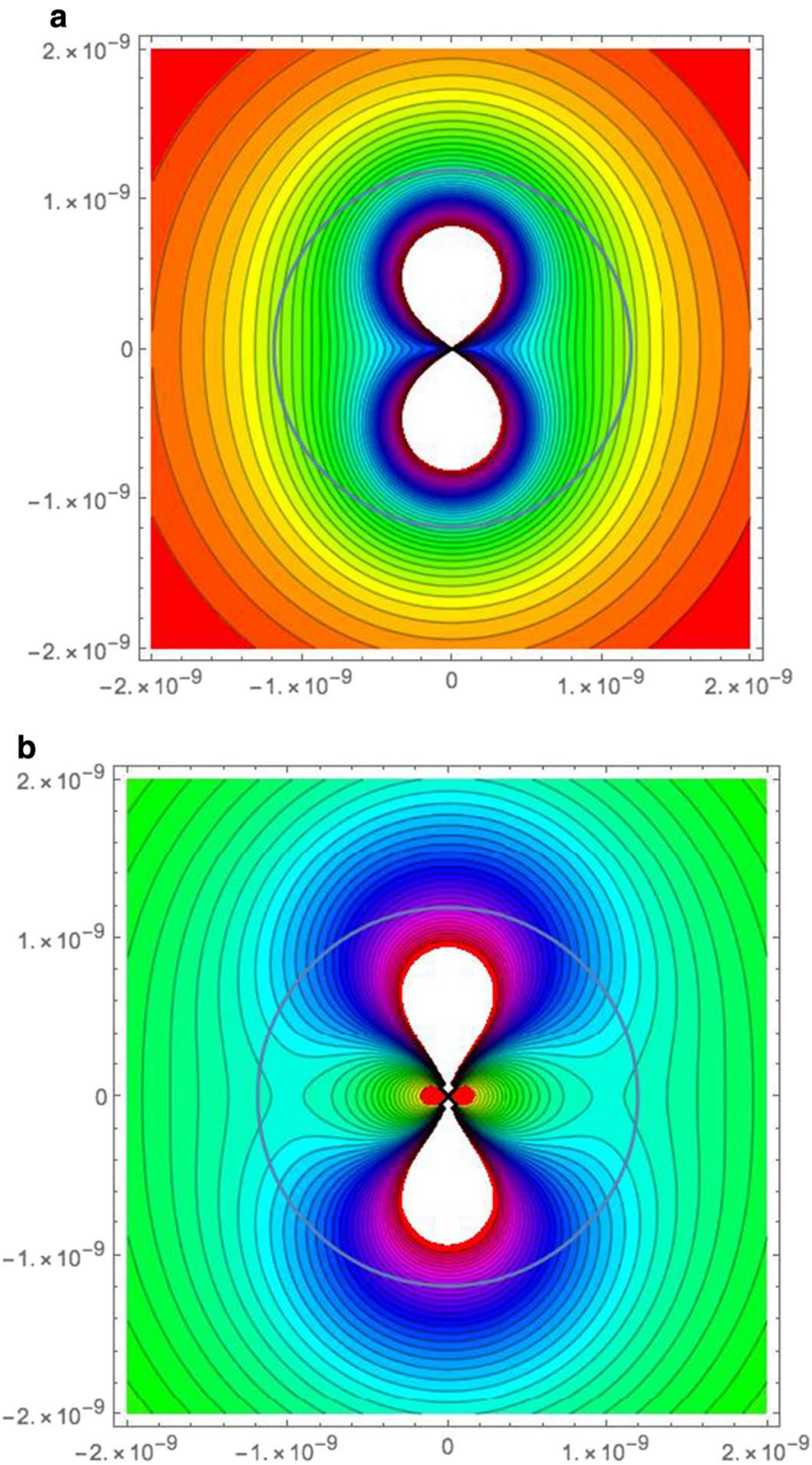

Fig. 6 Schematic representation (in arbitrary units) of pressure conditions around a black hole in an expanding, closed FLRW cosmos (the axis of rotation is vertical, the q component is on the top, the p components are shown below)

Since Fritz Zwickys discovery in the 1930s, it is known that the rotation velocity curves of the galaxies do not behave according Kepler's laws, if only the luminous matter is taken into account. These curves do not decline as a function of the distance from the center, but remain essentially flat. 


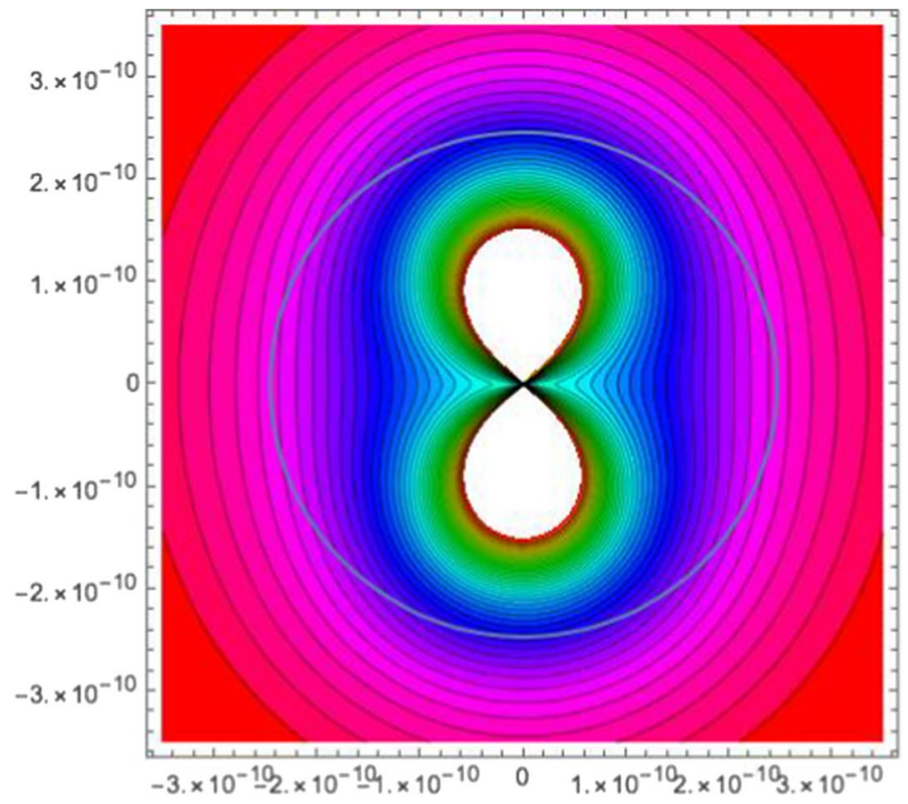

Fig. 7 Schematic representation (in arbitrary units) of the energy density $\rho$ around a black hole in an expanding cosmos (The axis of rotation is vertical)

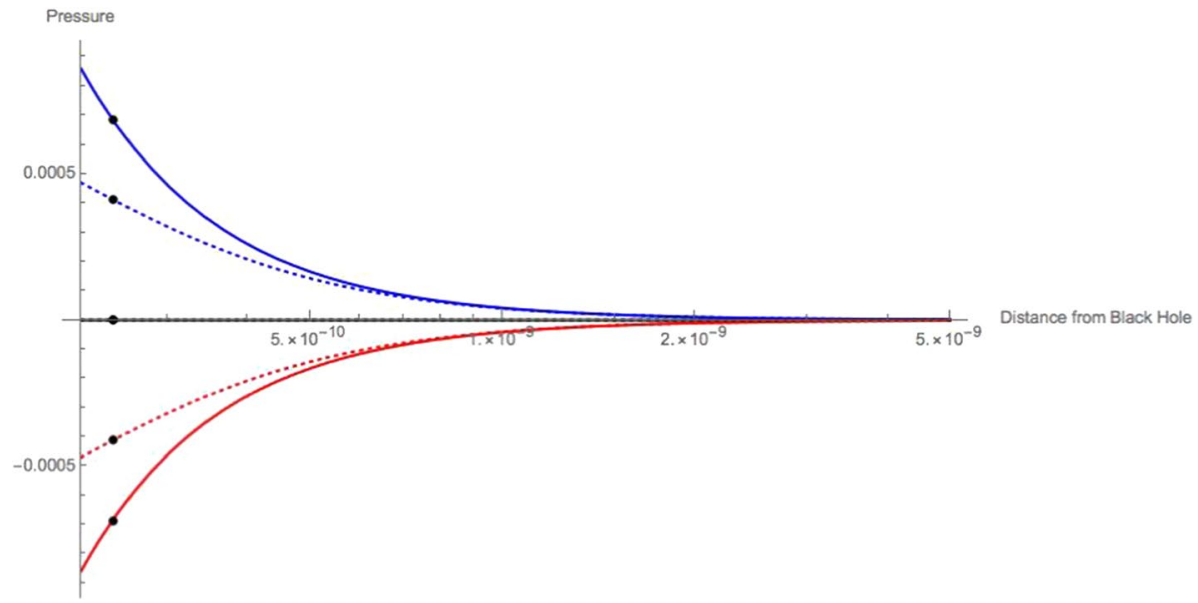

Fig. 8 Energy density (red), pressure components in the axis direction ( $q$ component-blue) and in the plane direction ( $p$ component-black), measured along the axis (drawn through) and in the plane (dotted). (Black dots mark the horizon.) The distance from the center is given in a logarithmic scale

In the immediate vicinity of a galactic center there are many stars around the black hole. The matter present there causes an approximately linear increase in the rotational velocity. It must be explained, however, why an almost constant rotational velocity can be observed in the outer regions of galaxies with little visible matter, and not a reduction of velocity, as would follow from Kepler's law. 


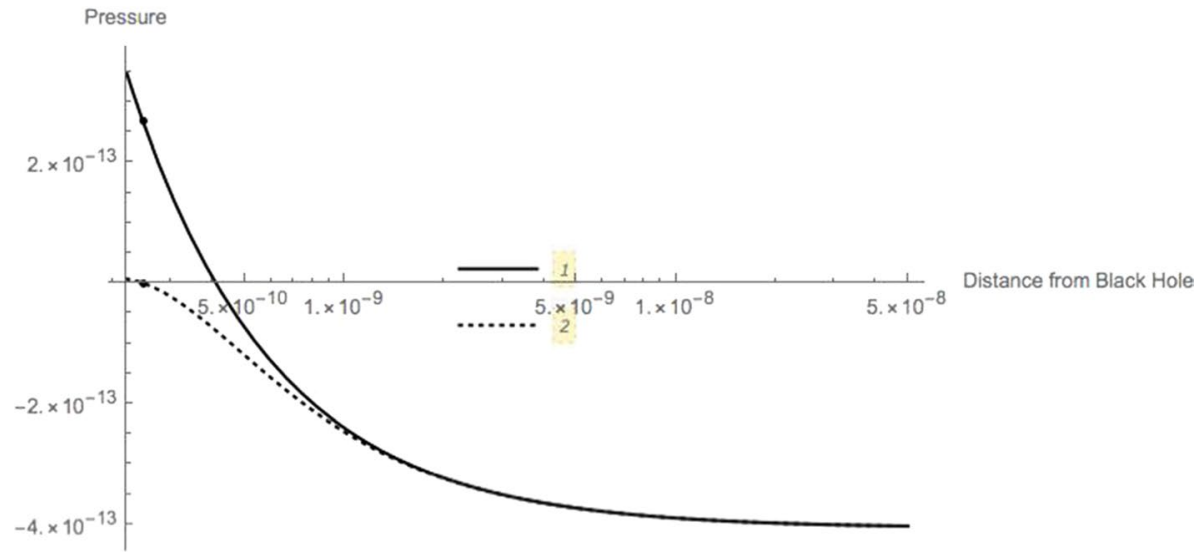

Fig. 9 The pressure of the $p$-component (1, measured along the axis, drawn through) and (2, measured in the plane, dotted) (the same units as in Fig. 8) (Note the logarithmic scale for the distances)

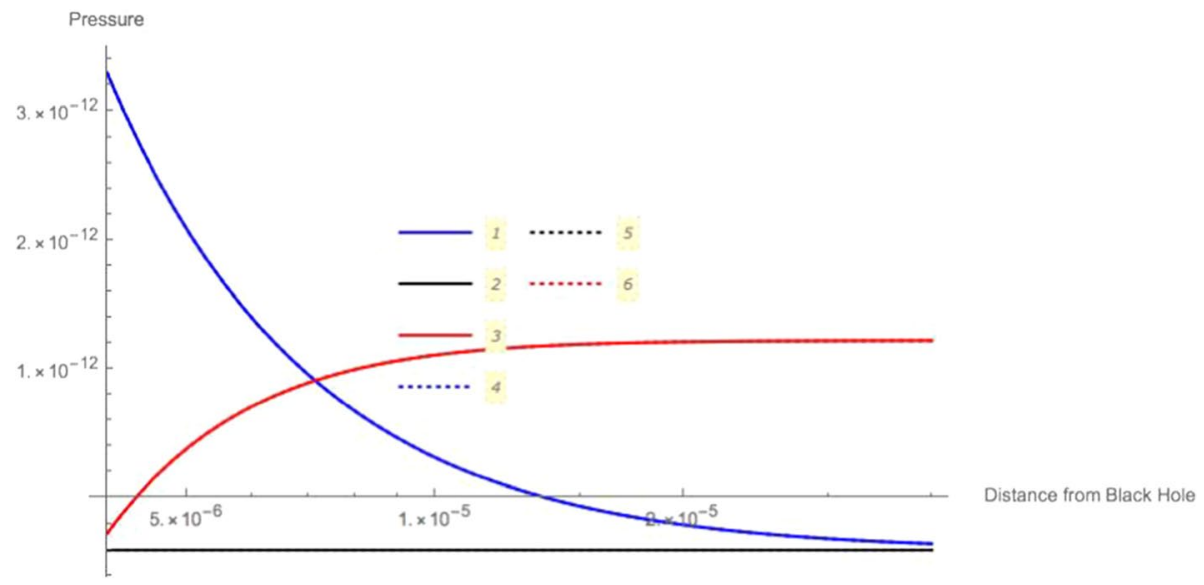

Fig. 10 The energy density $\rho$ (red) becomes positive and the pressure of the $q$ components (blue) became negative far from the horizon. There the $p$ components (black) are in a greater distance from the center equal to $-\rho / 3$ (Note the logarithmic scale for the distances)

A solution to this problem of the orbital velocity of galaxies comes from General Relativity $(G R T)$. For this, a negative pressure of the form $\boldsymbol{p}=-\rho / 3$ must be present in the cosmological model. Furthermore, it is still necessary that this negative pressure remains present near the horizon of the central Black Hole (Kerr solution).

In this case, two different effects seem to work together. The rotation of the Black Hole is reflected in the metric of the Kerr solution. Second, the negative cosmological pressure $\boldsymbol{p}=-\rho / 3$ changes the effect of gravity. As a result, flat velocity distributions are observed at the galaxies. The relevant papers with calculations of this effect are presented below.

The effect of the flat curves for the rotational velocities is an outcome of GRT, if there is a pressure $p_{\text {cosmic }}$ according to Eq. $(8-1)$ in the rotational plane of the black hole in its vicinity. 


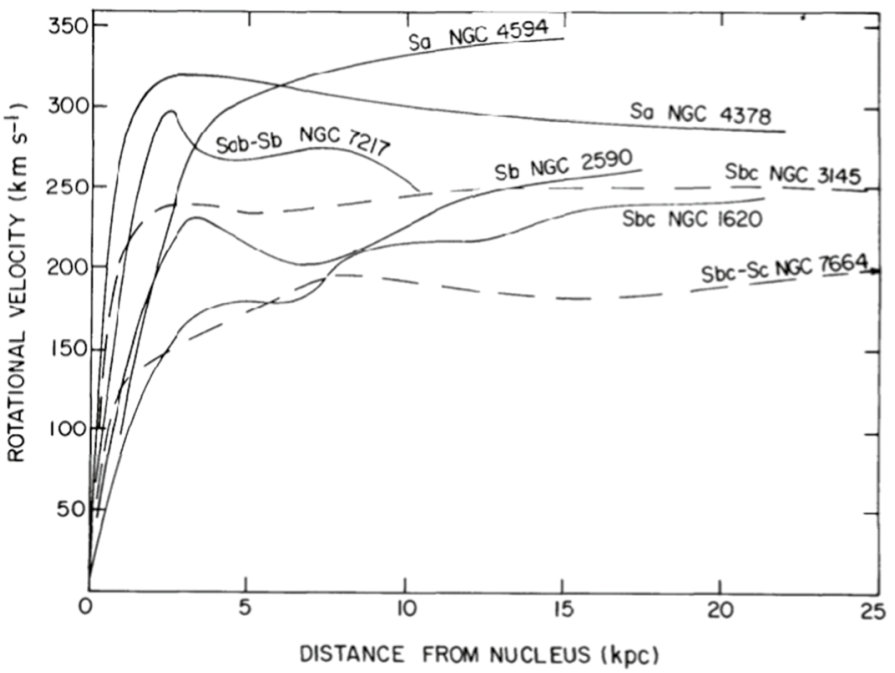

Fig. 11 Rotational velocities for seven galaxies, as a function of distance from the core. [Rubin et al. (1978)]

Fig. 12 Photo of the Hubble Space Telescope with Einstein Cross in the galaxy cluster MACS J1149.6+2223. (https:// hubblesite.org/contents/media/ images/2015/08/3496-Image. html)

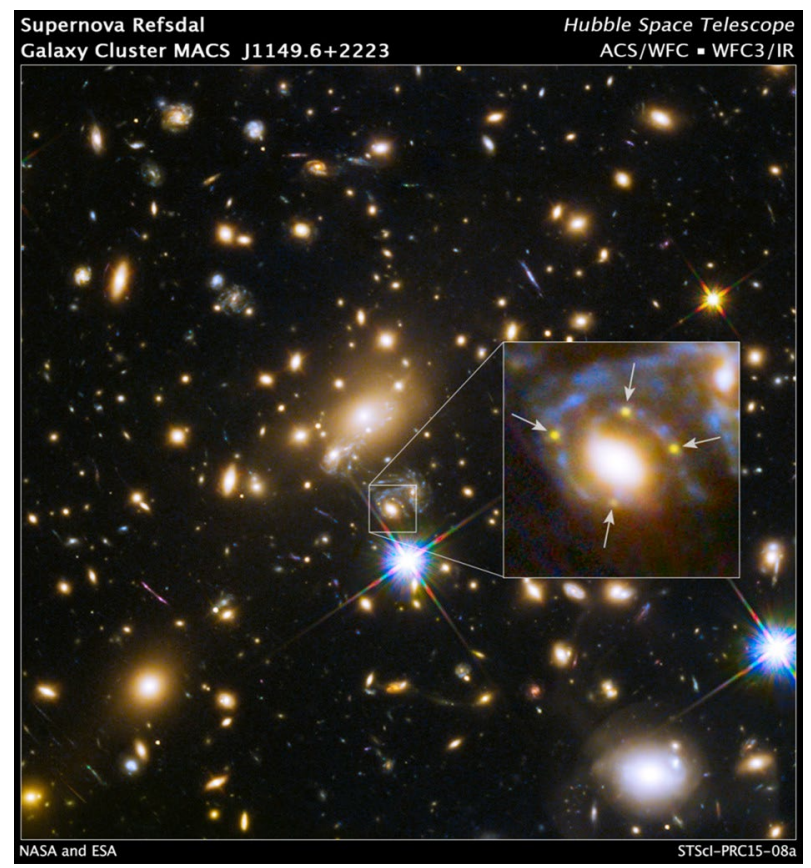

To our knowledge, this correlation with a negative pressure $\boldsymbol{p}=-\rho / 3$ was shown for the first time in papers by F. Siddhartha Guzmán and Tonatiuh Matos (2003) with Darío Núñez and Erandy Ramírez [Matos (2002)].

The authors postulate a hypothetical "Quintessence" with the state equation $\boldsymbol{p}=-\boldsymbol{\rho} / \mathbf{3}$ in the vicinity of a black hole. They show that such a pressure is the cause for the behaviour 
of rotation curves around the black hole, consistent with the observations at the outside regions of the galaxies.

However, they note [Matos (2002), p 297]:

»However, notice that in this approximation it cannot be said anything else about the matter producing the observed motion. The Newtonian approximation fixes the matter to be dust-perfect-fluid-like type. This is the usual way of reasoning: it is supposed a priori that the dark matter is a completely Newtonian dust and at the end of the day one arrives to a consistent description of the dark matter determining only the shape of the Newtonian gravitational potential.«

Recognizing Protyposis as the cosmological substrate, the change in the gravitational potential effected by cosmological pressure allows for the explanation sought by Guzmán, Matos et al. In other words, the Protyposis provides the properties of the unknown quintessence or matter postulated by Guzmán and Matos.

The approach by Guzmán and Matos was discussed by Valery Kiselev (2003), who corroborates the effects resulting for $p=-\rho / 3$, otherwise attributed to the hypothetical dark matter:

»Further, the angle surplus caused by the scalar field as found in [Matos (2000), Matos, Guzman, Urena-Lopez, Nunez (2001)] can be obtained in the perfect fluid approach used, too. Indeed, the limit of $\omega_{q} \rightarrow-1 / 3$ implies that the metric component $g_{r r}$ tends to a constant value.«

(Note that in quintessence models $\omega_{q}$ designates the ratio $p / \rho$.)

However, for the overall cosmology that is far from la black hole, Kiselev chooses other cosmological pressure values.

In interesting papers Rahaman et al. (2010) explore possible cosmological consequences. While we start from cosmology and describe the galactic consequences, these authors pursue an opposite approach: starting with the flat rotation curves of the galaxies and then considering cosmological consequences. Both approaches lead to the same equation of state:

$» \ldots$ the equation of state of 'curvature fluid' is found to be $\rho+3 p=0 \ldots$. «

Rahaman et al. assume a static cosmos, noting that this is only compatible in the form of a temporally local approximation. It is interesting that.

»... stable stellar orbits together with an attractive gravity exist only if $\omega_{q}$ is extremely close to $-1 / 3$, a result consistent with the special case studied by Guzman et al. (2003) Less exceptional forms of quintessence dark energy do not yield the desired stable orbits and are therefore unsuitable for modeling dark matter. ... Ordinarily quintessence dark energy is unable to do so: evidently, its repulsive action is too strong.« [Rahaman et al. (2011)]

Figure 9 show that in the rotational plane, $\omega_{q}$ is zero in the close vicinity of a black hole. However, in a somewhat greater distance the generally negative cosmological pressure $\boldsymbol{p}_{\text {cosmic }}=-\boldsymbol{\rho} / \mathbf{3}$ is reached very soon. Thus, the conditions are fulfilled so that in the galactic plane the visible matter circulates with constant velocity around the center in some larger distance. 


\section{Making the Rotational Velocities Plausible in a Newtonian Concept}

The most common explanation of constant orbital velocities outside the General Relativity Theory (GRT) is the postulate of "Dark Matter". Such an assumption is the easiest way in Newton's Theory of Gravity to explain an orbital velocity that does not change with the distance from the center of gravity. However, as already noted, the decades-long search for the necessary particles of Dark Matter has so far been unsuccessful. Therefore, it was obvious to look for other possible explanations.

Some authors [e.g. Begeman, (1991), Kroupa (2012), Kroupa (2015), Pawlowski (2020)], prefer "modified Newtonian dynamics" (MOND), invented by Milgrom (1994).

Their argumentation concerns astronomical observations in the Local Group, from which it follows that these findings lead to contradictions with the $\Lambda \mathrm{CDM}$ cosmology. [Kroupa (2012), Pawlowski (2020)].

\section{Rotational Velocities in the Protyposis Concept}

Going from the classical to a relativistic description, the tensor of energy density and pressure is relevant for the gravitational effect, rather than the mass density alone. The diagonalized energy density-pressure tensor has the form

$$
T_{i}^{k}=\left[\begin{array}{llll}
\rho & 0 & 0 & 0 \\
0 & p & 0 & 0 \\
0 & 0 & p & 0 \\
0 & 0 & 0 & p
\end{array}\right]
$$

As should be recalled, the pressure, [force/area], has the same dimension as the energy density [force $\times$ length/volume]. All four components of the tensor contribute equally to the gravitational effect.

While on earth and in laboratories pressures are in the range $0<p<\infty$, it has been recognized in recent years, even by the wider public, that pressure in GRT can also be negative.

A negative pressure behaves in cosmology as "anti-gravitation".

If the relation $3 p=-\rho$ holds for the pressure, the acceleration caused by the gravitative forces disappears in some sense. This became clear for the first time in cosmology with the cosmological constant and the "quintessence.

If $\boldsymbol{\rho}+\mathbf{3 p}>\mathbf{0}$, the gravitative effect predominates and the cosmos will recollapse after an expansion phase. That such an "end crash" would occur was the prevailing opinion 30 years ago, when the Protyposis cosmology was developed. Later that view was replaced by the idea of an accelerated expansion. To this effect, a cosmological constant and/or quintessence had to be postulated, so that $\boldsymbol{\rho}+\mathbf{3} \boldsymbol{p}<\boldsymbol{0}$ can be possible.

In the Protyposis cosmology, the state equation $\boldsymbol{\rho}+\mathbf{3 p}=\mathbf{0}$ is derived using very general quantum-theoretical and thermodynamical arguments.

Here, neither an acceleration nor deceleration effect arises in the cosmic expansion. Rather, as the result of adopting this state equation in the GRT, there is a cosmic expansion at the speed of light.

This cosmology solves many of the cosmological problems or makes pertinent artifices redundant, like dark energy, dark matter, the cosmological constant, the horizon problem and inflation, as well as the empiricism problem. 
As can be seen in Fig. 9, the gravitational effect of the galactic core is finally superseded by the cosmic effect of the Protyposis, so that, supposing an idealized situation, neither a considerable decline nor a considerable increase of the rotational velocity is to be expected.

The effect of the Protyposis thus brings about-without recourse to a hypothetic dark matter-the flat rotation velocity curves as calculated by Guzmán and Matos (2003).

\section{Conclusion}

These results can be interpreted as follows:

The Protyposis furnishes a mathematical model, describing essential cosmological observations on the basis of fundamental quantum theoretical properties, which does not need objects like particles of dark matter or dark energy.

Part of the AQIs of the Protyposis forms black holes as well as quantum particles [Görnitz, Graudenz, v. Weizsäcker (1992), Görnitz, Schomäcker (2012)]. Quantum fields can finally be described as an infinite amount of their field quanta and thus be produced from the particles.

From the cosmological perspective, the Protyposis acts like a liquid with the state equation $p=-\rho / 3$. The homogeneity and isotropy of the distribution of the cosmic Protyposis is locally modified by a rotating black hole, which allows for an explanation of the effects observed for the orbital speeds of the galaxies and gives a contribution to the creation of jet structures.

From the Protyposis model, flat rotation curves result due to the influence of cosmology, as calculated by Guzmán and Matos.

In addition, there are the gravitational effects of the large gravitation lenses, which can also be attributed to the effect of a dark matter, since they cannot be explained by visible matter alone. For example, they become visible at so-called Einstein Crosses. These Einstein Crosses appear when the light of a very far-away supernova runs on different ways around a centre of gravity, and then there are four images of a supernova.

Using the Protyposis, a hypothesis can also be formulated for the absence until now of hypothetical particles that shall form the dark matter:

Since the AQIs produce a gravitational effect, like everything in the cosmos, density fluctuations will become noticeable. Such density concentrations will presumably form especially around very early and very large black holes (for an analogy, see Fig. 4).

In such "clouds", however, where the energy density of the AQIs is higher than in their surroundings, the AQIs need not necessarily form particles.

The Protyposis cosmology model started from the assumption of a homogenous and isotropic distribution of energy density and cosmological pressure, whereas quantum theory suggests that there are local density fluctuations. Areas with a higher energy density will produce a stronger gravitational effect.

The complicated effects of Protyposis inhomogeneities on the curvature of light rays are beyond the scope of our toy model. For this purpose, high-performance computer codes for of the numerical treatment of the GRT equations are required, which were not at our disposal.

75 years ago, Einstein pleaded for an algebraic, i.e., discrete structure for the foundation of physics. Today it has become plain how pertinent and productive this call has been. However, here it was necessary to abandon the notion of quantum fields constructed from particles as field quanta as the fundamental ultimate entities. 
Of course, the idea of particles, objects that can freely be moved in space and time while maintaining their 'identity', is highly productive and useful. Yet, it is inherent to the notion of particles, that ever smaller particles can be contrived-or particles having a still simpler sub-structure. By contrast, the quantum bit is a structure to which there cannot be a simpler one.

Concomitantly, though, "almost no information" means no localization. Thus, a quantum bit as "the simplest" entity has to be perceived as being a cosmic structure.

Acknowledgements We thank Jochen Schirmer, Sebastian Stier and Pavel Kroupa for valuable hints and comments, as well as the referees for helpful remarks.

Author contributions T. G. developed the protyposis concept, U. S. carried out the mathematica ${ }^{\circledR}$ computations.

Funding Open Access funding enabled and organized by Projekt DEAL.

\section{Declarations}

Conflict of interest The authors declare that the research was conducted in the absence of any commercial or financial relationships that could be construed as a potential conflict of interest.

Open Access This article is licensed under a Creative Commons Attribution 4.0 International License, which permits use, sharing, adaptation, distribution and reproduction in any medium or format, as long as you give appropriate credit to the original author(s) and the source, provide a link to the Creative Commons licence, and indicate if changes were made. The images or other third party material in this article are included in the article's Creative Commons licence, unless indicated otherwise in a credit line to the material. If material is not included in the article's Creative Commons licence and your intended use is not permitted by statutory regulation or exceeds the permitted use, you will need to obtain permission directly from the copyright holder. To view a copy of this licence, visit http://creativecommons.org/licenses/by/4.0/.

\section{References}

Aghanim N., et al. [Planck Collaboration], Planck 2018 results. VI. Cosmological parameters, arxiv e-prints, (2018) arXiv:1807.06209.

Aghanim N. et al. [Planck Collaboration], Planck 2018 results. V. CMB power spectra and likelihoods, arxiv e-prints, arXiv:1907.12875 [astro-ph.CO].

Arndt, M., \& Hornberger, K. (2014). Testing the limits of quantum mechanical superpositions. Nature Physics, $10,271$.

Beacham, J., de Roeck, A. and Lanfranchi, G. (2020) ALICE'S DARK SIDE, CERN-Courier, 2020 NovDec, p. $25-27$

Begeman, K. G., Broeils, A. H., \& Sanders, R. H. (1991). Extended rotation curves of spiral galaxies: Dark haloes and modified dynamics. MNRAS, 249, 523.

Chen, W., \& Wu, Y.J.-S. (1990). Implications of a cosmological constant varying as $\mathrm{R}^{-2}$. Phys Rev D, 41, 695-697.

Cirelli, M. et al. (2012) PPPC 4 DM ID: A Poor Particle Physicist Cookbook for Dark Matter Indirect Detection, CERN-PH-TH/2010-057 SACLAY \{T10/025 IFUP-TH/2010-44, https://arxiv.org/abs/1012.4515 [hep-ph] 21 Aug 2012

Colin, J., Mohayaee, R., Rameez, M., \& Sarkar, S. (2019). Evidence for anisotropy of cosmic acceleration. $A \& A, 631, \mathrm{~L} 13$.

DiValentino, E., Melchiorri, A., Silk, J. (2020) Planck evidence for a closed Universe and a possible crisis for cosmology, Nature Astronomy 196-203

Einstein, A. (1954). The Meaning of Relativity (6th ed., p. 164). Princeton University Press Princeton, New Jersey.

Finkelstein, D. (1969). Space-Time Code. Physical Review, 184, 1261-1271. 
Gooth, J. et al. (2019) Axionic charge-density wave in the Weyl semimetal ( $\left.\mathrm{TaSe}_{4}\right)_{2} \mathrm{I}$ Nature https://doi.org/ 10.1038/s41586-019-1630-4

Görnitz, T. (1986). New Look at the Large Numbers. Intern Journ Theoret Phys., 25, 897-905.

Görnitz, T. (1988a). Abstract quantum theory and space-time structure, part I, Ur theory, space-time continuum and Bekenstein-Hawking entropy. Intern Journ Theoret Phys, 27, 527-542.

Görnitz, T. (1988b). On connections between abstract quantum theory and space-time-structure, part II, A model of cosmological evolution. Intern Journ Theoret Phys, 27, 659-666.

Görnitz, T. (2011). Deriving general relativity from reflections on quant. Information, Adv Sci Lett, 4, $577-585$.

Görnitz, T. (2013). What happens inside a black hole? Quantum Matter, 2, 21-24.

Görnitz, T. (2014). Simplest quantum structures and the foundation of interaction. Rev Theor Sci, 2, 289-300.

Görnitz, T., Graudenz, D., Weizsäcker, C. F., \& v. . (1992). Quantum field theory of binary alternatives. Intern J Theoret Phys, 31, 1929-1959.

Görnitz, T., \& Ruhnau, E. (1989). Connections between abstract quantum theory and space-time-structure, part III. Vacuum Structure and Black Holes, Intern Journ Theoret Phys, 28, 651-657.

Görnitz, T., \& Schomäcker, U. (2012). Quantum particles from quantum information. Journal of Physics: Conference Series, 380, 012025. https://doi.org/10.1088/1742-6596/380/1/012025

Görnitz, T., \& Schomäcker, U. (2016). The structures of interactions - how to explain the gauge groups U(1), SU(2) and SU(3). Foundations of Science. https://doi.org/10.1007/s10699-016-9507-6

Görnitz, T., Weizsäcker, C. F. v. (1986), De Sitter representations and the particle concept in an ur-theoretical cosmological model, in Conformal Groups and Related Symmetries, Physical Results and Mathematical Background, A. O. Barut, H. D. Doebner, eds, Springer Berlin

Guzmán, F. S., Matos, T. (2003) Quintessence-like Dark Matter in Spiral Galaxies, Rev. Mex. F`is. 49 (3) 203-206

Hawking, S. W., \& Ellis, G. F. R. (1973). The large scale structure of space-time. University Press London.

Ijjas, A., Steinhardt, P. J., \& Loeb, A. (2017). POP goes the universe - the latest astrophysical measurements, combined with theoretical problems, cast doubt on the long-cherished inflationary theory of the early cosmos and suggest we need new ideas. Scientific American, 1, 32.

Ivanov M M, Simonovic M, Zaldarriaga M (2020) Cosmological Parameters from the BOSS Galaxy Power Spectrum, https://arxiv.org/abs/1909.05277 [astro-ph.CO] 27 May 2020,

Jacobson, T. (1995). Thermodynamics of Spacetime: The Einstein Equation of State. Physical Review Letters, 75,1260 .

John, M. V. (2019). $\mathrm{R}_{\mathrm{h}}=\mathrm{ct}$ and the eternal coasting cosmological model. MNRAS Letters, 484(1), L35-L37.

John, M. V., \& Joseph, K. B. A. (1996). Modified Ozer-Taha type cosmological model. Physics Letters B, 387, 466-470.

John, M. V., Joseph, K. B. (2000) Generalised Chen-Wu type cosmological model, Physical Review D 61, 087304

John, M. V., Narlikar, J. V. (2002) Comparison of cosmological models using Bayesian theory, Phys Rev D 65, 043506

Kiselev, V. V. (2003). Quintessence and black holes. Class. Quantum Grav., 20, 1187-1197.

Weizsäcker, C. F. v. (1958) Komplementarität und Logik II, Z. f. Naturforschung (1958) 13a, 245

Kroupa, P. (2012). The dark matter crisis: Falsification of the current standard model of cosmology. Publications of the Astronomical Society of Australia, 29, 395-433.

Kroupa, P. (2015) Galaxies as simple dynamical systems: observational data disfavor dark matter and stochastic star formation, CaJPh. 93.169K; https://arxiv.org/abs/1406.4860

López-Corredoira, M., Melia, F., Lusso, F. E., \& Risaliti, G. (2016). Cosmological Tests with the QSO Hubble Diagram. Intern. Journal of Modern Physics D, 25, 1650060.

Matos, T., Guzman, F. S., Nunez, D. (2000) Spherical Scalar Field Halo in Galaxies, Phys. Rev. D 62, 061301

Matos, T., Guzman, F. S., Urena-Lopez, L. A., Nunez, D. (2001) Scalar Field Dark Matter, astro-ph/0102419

Matos, T., Núñez, D., Guzmán, F. S., \& Ramírez, E. (2002). Geometric Conditions on the Type of Matter Determining the Flat Behavior of the Rotational Curves in Galaxies. General Relativity and Gravitation, 34, 283-305.

Melia, F. (2016). Definitive test of the $\mathrm{R}_{\mathrm{h}}=$ ct universe using redshift drift. MNRAS, 463, L61-L63.

Melia, F. (2017). The zero active mass condition in Friedmann-Robertson-Walker cosmologies. Frontiers of Physics, 12(1), 129802. https://doi.org/10.1007/s11467-016-0611-4

Melia, F., \& López-Corredoira, M. (2017). Alcock-Paczynski Test with Model-independent BAO Data, International Journal of Modern Physics D, 26. No., 06, 1750055.

Melia, F., \& Maier, R. S. (2013). Cosmic Chronometers in the $\mathrm{R}_{\mathrm{h}}=\mathrm{ct}$ Universe. MNRAS, 432, 2669. 
Melia, F., \& McClintock, T. M. (2015). Supermassive Black Holes in the Early Universe. Proceedings of the Royal Society A, 471, 20150449.

Melia, F., \& Shevchuk, A. S. H. (2012). The $\mathrm{R}_{\mathrm{h}}=$ ct universe. MNRAS, 419, 2579.

Milgrom, M. (1994). Dynamics with a non-standard inertia-acceleration relation: An alternative to dark matter. Annalen Der Physik, 229, 384-415.

Mortlock, D. J., et al. (2011). A luminous quasar at a redshift of z = 7.085. Nature., 474(7353), 616-9. https://doi.org/10.1038/nature 10159

Pan, J.-W., et al. (2017). Satellite-based entanglement distribution over 1200 kilometers. Science, 356(6343), 1140-1144.

Pawlowski, M. S., \& Kroupa, P. (2020). The Milky Way's disc of classical satellite galaxies in light of Gaia DR2. MNRAS., 491, 3042P.

Rahaman, F., Kuhfittig, P. K. F., Chakraborty, K., Kalam, K., \& Hossain, D. (2011). Modelling galactic halos with predominantly quintessential matter. International Journal of Theoretical Physics, 50, 2655-2665.

Rahaman, F., Nandi, K. K., Bhadra, A., Kalam, M., \& Chakraborty, K. (2010). Perfect fluid dark matter. Physics Letters B, 694, 10-15.

Raveri, M. (2020) Reconstructing gravity on cosmological scales, Phys Rev D 101, 083524

Rubin, V. C., Ford, W. K., \& Thonnard, N. (1978). Extended rotation curves of high-luminosity spiral galaxies. IV. Systematic Dynamical Properties, Astrophysical Journal, 225, L107-L111.

Scheibe, E., Süssmann, G., Weizsäcker, C. F. v. (1958) Mehrfache Quantelung, Komplementarität und Logik III, Z. f. Naturforschung 13a, 705

Smith, A., \& Bromm, V. (2019). Supermassive black holes in the early universe. Contemporary Physics. https://doi.org/10.1080/00107514.2019.1615715

Steinhardt, P. J. (2014). Big Bang blunder bursts the multiverse bubble. Nature, 510, 9.

Steinhardt, P. L., et al. (2016). The impossibly early galaxy problem. The Astrophysical Journal, 824, 21.

Vaidya, P. C. (1977). The Kerr metric in cosmological background, Pramãna, 8. Nr., 6, 512-517.

Verlinde, E. (2011) On the origin of gravity and the laws of Newton, Journal of High Energy Physics. 04, Nr. 29

Wei J.-J., Wu X.-F., Melia, F., Maier, R. S. (2015) The Gamma-ray Burst Hubble Diagram and its Cosmological Implications, The Astrophysical Journal, 772, id 43

Weizsäcker, C. F. v. (1955) Komplementarität und Logik I. Naturwissenschaft, 42(521-529), 545-555.

Weizsäcker, C. F. v. (1971) Die Einheit der Natur. Hanser, München, Engl.Ed. (1980) The unity of nature, Farrar Straus Giroux; New York

Weizsäcker, C. F. v. (1985) Aufbau der Physik, Hanser München, (engl. Edition: Weizsäcker C F v. The Structure of Physics, edited, revised and enlarged by Görnitz, T. \& Lyre, H. (2006) Springer, Heidelberg)

Wheeler, J. A. (1989) INFORMATION, PHYSICS, QUANTUM: THE SEARCH FOR LINKS, in Proc. 3rd Int. Symp. Foundations of Quantum Mechanics, Tokyo pp. 354-368

Yennapureddya, M. K., \& Melia, F. (2018). A cosmological solution to the Impossibly Early Galaxy Problem. Physics of the Dark Universe, 20, 65-71. https://doi.org/10.1016/j.dark.2018.03.003

Publisher's Note Springer Nature remains neutral with regard to jurisdictional claims in published maps and institutional affiliations.

Thomas Görnitz was Professor for Didactics of Physics at the Goethe Univ. in Frankfurt/M. He was winner oft the GDR-Mathematics Olympics and studied physics and mathematics in Leipzig, receiving a PhD in mathematical physics. After applying for permission to leave the communist country, he worked in the GDR as a gravedigger. He was finally able to settle in Munich with his family. Carl Friedrich v. Weizsäcker won him for cooperation in fundamental questions of quantum physics and cosmology, which finally lasted over two decades. Afterwards, he worked on the Inst. for Mathematical Physics at the TU Braunschweig. He works on the implications of quantum theory for understanding the universe and its evolutionary processes. Publications include: Quanta Are Different (1999), Protyposis - an introduction/ Consciousness and Matter from Quantum Information (2018) and (with Dr. Brigitte Görnitz) The Creative Cosmos: Mind and Matter from Quantum Information(2002), From Quantum Theory to Consciousness - Cosmos, Mind, Matter (2016).

Uwe Schomäcker has worked in the field of IT development for finance systems already during his study of physics. After his Diploma, he joined the scientific team of Carl Friedrich v. Weizsäcker for working on 
the Urtheory. With his $\mathrm{PhD}$ in physics, he became assistant professor at TU Braunschweig, holding lectures to date for modelling complex systems in computational physics. In parallel, he has become co-founder of several companies over the years, working, among others, in the fields of IT technology consultancy and software development for logistics and finance applications; consultancy in researching market data, trends, and developments of the global air transport community. 